\title{
Zur Theorie der Verteilungen von Potential, elektrischer Feldstärke und Stromdichte in einem Lichtbogen endlicher Länge im starken axialen Magnetfeld
}

\author{
J. RAEDER und S. WirTZ \\ Institut für Plasmaphysik, Garching bei München \\ (Z. Naturforsch. 24 a, 1433-1448 [1969] ; eingegangen am 24. Juni 1969)
}

\begin{abstract}
A partial differential equation for the electric potential in an arc with an applied axial magnetic field is derived by using Ohm's law and the equations $\nabla \cdot \boldsymbol{j}=0$ and $\nabla \times \boldsymbol{E}=0$. To clarify the physics the potential equation is solved for two simple cases where the plasma is assumed to be homogeneous. The solutions reveal that the Hall parameter $\omega_{\mathrm{e}} \tau_{\mathrm{e}}$ and the arc length strongly influence the potential distribution. The dependence of the potential on the axial and radial temperature profiles is studied numerically by relaxation methods.
\end{abstract}

Zur Erzeugung stationärer Wasserstoffplasmen hoher Temperatur haben sich elektrische Lichtbögen bewährt, denen ein starkes axiales Magnetfeld überlagert wird. Dieses reduziert die radialen Verluste durch Wärmeleitung so stark, daß bei Magnetfeldern von 1 bis 4 Tesla und einem Bogenstrom von etwa 2000 A Achsentemperaturen von $1-2 \cdot 10^{5}{ }^{\circ} \mathrm{K}$ erreicht wurden ${ }^{1,2}$. Die Abb. 1 zeigt eine schematische Darstellung der von Zankl verwendeten Apparatur. Der Bogen brennt zwischen massiven Elektroden aus

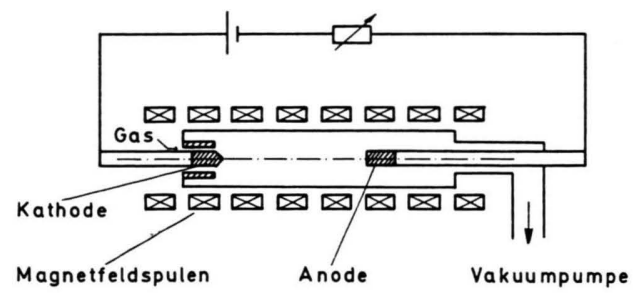

Abb. 1. Schema der experimentellen Anordnung.

Wolfram von etwa $2 \mathrm{~cm}$ Durchmesser. Da die Entladung bei vermindertem Druck betrieben wird - der Druck außerhalb der heißen Zone beträgt ungefähr 8 Torr - umschließt ein zylindrischer Glaskessel die gesamte Anordnung. Die lichte Weite dieses Kessels ist wesentlich größer als der Kathodendurchmesser. Die visuelle Feststellung einer Bogenentladung von ca. $3 \mathrm{~cm}$ Durchmesser wird durch die Messung der radialen Temperaturverteilung bestätigt $^{2}$. Mit der bekannten Theorie für zylindersymmetrische, wandstabilisierte Bögen, d. h. für unendlich lange Bögen, gelingt eine mit den Messun-

Sonderdruckanforderungen erbeten an Dr. J. RAEDER, Institut für Plasmaphysik, D-8046 Garching b. München.

1 C. Mahn, H. Ringler u. G. Zankl, Z. Naturforsch. 23 a, 867 [1968]. gen übereinstimmende Berechnung des Temperaturprofils nur dann, wenn man an Stelle des Rohrdurchmessers den optisch sichtbaren Durchmesser des Bogens einsetzt ${ }^{3}$. Dieses Ergebnis ist unbefriedigend, da erst der Glaskessel die Wand mit bekannter Temperatur bildet. Ihre Verwendung in der Randbedingung bei der Lösung der Energiebilanz führt jedoch zu einer Temperaturverteilung, die wesentlich zu breit ist. Dieser Widerspruch zum Experiment zeigt, da $\beta$ der Bogen nicht nur von der Wand stabilisiert wird, sondern auch noch durch andere Mechanismen.

Die Beschränkung der heißen Zone auf einen kleinen Durchmesser zeigt, daß der Bogen auch nur in diesem schmalen Bereich geheizt wird, daß also die axiale elektrische Feldstärke außerhalb des leuchtenden Zylinders klein ist. Dieser Befund ist leicht qualitativ zu erklären. Die den Strom transportierenden Elektronen geraten nach ihrer Emission aus der Kathode unter den Einfluß des starken Magnetfeldes, das sie während der Drift zur Anode auf eine spiralförmige Bahn zwingt. Wenn der Larmor-Radius wesentlich kleiner ist als die mittlere freie Weglänge der Elektronen, d. h. wenn der Hall-Parameter $\omega_{\mathrm{e}} \tau_{\mathrm{e}}$ wesentlich größer als 1 ist, bleibt der elektrische Strom praktisch auf das Innere der magnetischen Flußröhre beschränkt, die gerade die Mantelfläche der Kathode umschließt. Makroskopisch wird andererseits das Verhalten der Elektronen durch das Ohmsche Gesetz beschrieben, nach dem die axiale Stromdichte im wesentlichen dem elektrischen Feld in dieser Richtung proportional ist. Das mikroskopische Bild stimmt nur dann mit dem makroskopi-

2 G. ZANKL, Veröffentlichung in Vorbereitung.

3 U. Heidrich, Z. Naturforsch. 20 a, 475 [1965]. 
schen überein, wenn die axiale elektrische Feldstärke außerhalb der beschriebenen magnetischen Flußröhre stark abklingt. Wegen der Rotationsfreiheit des elektrischen Feldes entstehen dort radiale, elektrische Felder, die aber nur kleine Ströme verursachen, da die Bewegung der Elektronen quer zum Magnetfeld stark gehemmt wird. Die Existenz des durch die Magnetisierung stark anisotropen Plasmas zwischen den Elektroden führt also zu einer starken Verzerrung des Vakuumfeldes im Sinne einer Konzentration der axialen elektrischen Feldstärke um die Achse der Anordnung. Die Annahme eines konstanten elektrischen Feldes, das aus exakter Zylindersymmetrie folgen würde, ist also nicht mehr zulässig, eine darauf aufbauende Theorie kann die Temperaturverteilung nicht richtig bestimmen. Um die Grundlagen zu deren Berechnung im stark magnetisierten, rotationssymmetrischen Plasma endlicher Länge zu schaffen, sollen die Verteilungen des elektrischen Potentials für verschiedene Modelle in dieser Arbeit berechnet werden.

\section{Grundgleichungen und Annahmen}

Da die stationäre Verteilung des elektrischen Potentials aus der Bedingung $\nabla \cdot \boldsymbol{j}=0(\boldsymbol{j}=$ elektrische Stromdichte) bestimmt wird, soll zunächst das verwendete Ohmsche Gesetz angegeben und diskutiert werden. Wir benutzen die Form der Stromtransportgleichung, die von BRAGINSKY ${ }^{4}$ für ein vollionisiertes Zweikomponentenplasma im lokalen thermodynamischen Gleichgewicht abgeleitet wurde:

$$
\boldsymbol{E}^{\prime}=\frac{\boldsymbol{j}_{\|}}{\sigma_{\|}}+\frac{\boldsymbol{j}_{\perp}}{\sigma_{\perp}}+\frac{1}{\sigma_{\times}} \boldsymbol{j} \times \boldsymbol{h}+\frac{1}{e n_{\mathrm{e}}} \boldsymbol{j} \times \boldsymbol{B}
$$

mit

$$
\begin{aligned}
& \boldsymbol{E}^{\prime}=\boldsymbol{E}+\boldsymbol{v} \times \boldsymbol{B}+\frac{1}{e n_{\mathrm{e}}} \nabla p_{\mathrm{e}} \\
& +\frac{1}{e n_{\mathrm{e}}}\left(\beta_{\|} \nabla_{\|} T+\beta_{\perp} \nabla_{\perp} T+\beta_{\times} \boldsymbol{h} \times \nabla T\right)
\end{aligned}
$$$$
(\boldsymbol{h}=\boldsymbol{B} / B) \text {. }
$$

Die Vektoren $\boldsymbol{j}_{\|}, \boldsymbol{j}_{\perp}$ und $\nabla_{\|} T, \nabla_{\perp} T$ sind die Komponenten von $\boldsymbol{j}$ und $\nabla T$ parallel bzw. senkrecht zum Magnetfeld B. Die Koeffizienten $\beta_{\perp}$ und $\beta_{\times}$ sind Funktionen von $T, n_{\mathrm{e}}$ und $B ; \beta_{\|}$hängt nicht von $B \mathrm{ab}$, da es einen Transportvorgang parallel zu $\boldsymbol{B}$ beschreibt. Die beiden Leitfähigkeitskoeffizien-

4 S.I.Braginsky, in: M.A.Leontovich, Reviews of Plasma Physics, Consultants Bureau, New York 1967, Band 1. ten $\sigma_{\perp}$ und $\sigma_{\|}$folgen für großes $B$ den Gleichungen ${ }^{4}$

$$
\begin{aligned}
\sigma_{\perp} & =e^{2} n_{\mathrm{e}} \tau_{\mathrm{e}} / m_{\mathrm{e}}, \\
\sigma_{\|} & =1,95 \sigma_{\perp} .
\end{aligned}
$$

Im vollionisierten Plasma ist $\tau_{\mathrm{e}}=\tau_{\mathrm{ei}}$ eine Stoßzeit für Impulsaustausch zwischen Elektronen und Ionen. Sie ist so bestimmt, daß die Wechselwirkung von Elektronen, deren Maxwell-Verteilung um die Relativgeschwindigkeit $\boldsymbol{u}$ gegen die Geschwindigkeitsverteilung der Ionen verschoben ist, zu der Reibungskraft pro Volumeinheit $\boldsymbol{R}=-\left(m_{\mathrm{e}} n_{\mathrm{e}} / \tau_{\mathrm{ei}}\right) \boldsymbol{u}$ führt (ohne numerische Koeffizienten). Parallel zu $\boldsymbol{B}$ gilt diese einfache Formel für die Reibungskraft nicht, da ein elektrisches Feld in dieser Richtung die Geschwindigkeitsverteilung der Elektronen zugunsten der schnellen Elektronen deformiert. Der Grund hierfür ist die starke Abnahme des Coulomb-Querschnittes mit der Geschwindigkeit $\left(\propto 1 / v^{4}\right)$. Senkrecht zu einem starken Magnetfeld tritt dieser Effekt nicht auf, da hier die Elektronen nur über den kleinen Gyrationsradius beschleunigt werden können. Deshalb ist $\sigma_{\|}$bei Stößen von Elektronen mit einfach geladenen Ionen um den Faktor 1,95 größer als $\sigma_{\perp}$; für $B=0$ gilt natürlich $\sigma_{\|}=\sigma_{\perp}=\sigma$. Außerdem tritt in Gl. (1) noch der Koeffizient $\sigma_{\times}$auf, da das Magnetfeld auch eine Reibungskraft proportional zu $\boldsymbol{u} \times \boldsymbol{h}$ verursacht.

Während $\sigma_{\|}$der Proportionalitätsfaktor zwischen $\boldsymbol{j}_{\|}$und $\boldsymbol{E}_{\|}$ist, stellt $\sigma_{\perp}$ den Porportionalitätsfaktor zwischen $\boldsymbol{j}_{\perp}$ und $\boldsymbol{E}_{\perp p}^{\prime}$ dar $\left(\boldsymbol{E}_{\perp \text { p }}^{\prime}\right.$ ist die Projektion von $\boldsymbol{E}_{\perp}^{\prime}$ auf $\left.\boldsymbol{j}_{\perp}\right)$ :

$$
\begin{aligned}
& \boldsymbol{E}_{\perp \mathrm{p}}^{\prime}=\frac{\left(\boldsymbol{E}_{\perp}^{\prime} \cdot \boldsymbol{j}_{\perp}\right) \boldsymbol{j}_{\perp}}{j_{\perp}^{2}}=\frac{\boldsymbol{j}_{\perp}}{\sigma_{\perp}} \\
& +\left(\frac{1}{\sigma_{\times}}+\frac{B}{e n_{\mathrm{e}}}\right) \frac{\left[(\boldsymbol{j} \times \boldsymbol{h}) \cdot \boldsymbol{j}_{\perp}\right] \boldsymbol{j}_{\perp}}{j_{\perp}^{2}}=\frac{\boldsymbol{j}_{\perp}}{\sigma_{\perp}} .
\end{aligned}
$$

Wenn $B$ sehr groß ist, steht $\boldsymbol{E}_{\perp}^{\prime}$ nahezu senkrecht auf $\boldsymbol{j}_{\perp}$, da die Terme proportional zu $\boldsymbol{j} \times \boldsymbol{B}$ in Gl. (1) dominieren. Das zur Erzeugung einer bestimmten Stromdichte $\boldsymbol{j}_{\perp}$ notwendige Feld $\boldsymbol{E}_{\perp}^{\prime}$ steigt mit $B$ an, da zwar wegen der Gln. (3) und (5) $\boldsymbol{E}^{\prime}{ }_{\perp \text { p }}$ konstant bleibt, der Winkel zwischen $\boldsymbol{E}_{\perp}^{\prime}$ und $\boldsymbol{E}_{\perp \text { p }}^{\prime}$ aber mit $B$ gegen $90^{\circ}$ wächst.

Für die folgenden Rechnungen ist es günstiger, das Ohmsche Gesetz (1) nach $\boldsymbol{j}$ aufzulösen:

$$
\begin{aligned}
\boldsymbol{j}=\sigma_{1} \boldsymbol{E}_{\|}^{*} & +\sigma_{2} \boldsymbol{E}_{\perp}^{*}+\sigma_{3} \boldsymbol{E}^{*} \times \boldsymbol{h}+\beta_{1} \nabla_{\|} \boldsymbol{T} \\
& +\beta_{2} \nabla_{\perp} T+\beta_{3} \nabla \boldsymbol{T} \times \boldsymbol{h} \\
\mathrm{mit} \quad \boldsymbol{E}^{*}= & \boldsymbol{E}+\boldsymbol{v} \times \boldsymbol{B}+\left(1 / e n_{\mathrm{e}}\right) \nabla p_{\mathrm{e}}
\end{aligned}
$$


und

$$
\sigma_{1}=\sigma_{\|} .
$$

Die Koeffizienten $\sigma_{2}, \sigma_{3}, \beta_{1}, \beta_{2}$ und $\beta_{3}$ lassen sich durch $\sigma_{1}$ und den Hall-Parameter $x=\omega_{\mathrm{e}} \tau_{\mathrm{e}}\left(\omega_{\mathrm{e}}=\right.$ $\left.e B / m_{\mathrm{e}}\right)$ ausdrücken. Formeln für das Ohmsche Gesetz sind im Anhang zusammengestellt.

Wir wollen das Ohmsche Gesetz (6) auch auf die Außenbereiche des Lichtbogens anwenden, in denen das Gas nur teilweise ionisiert ist. Zwar liegen für teilweise ionisierten Wasserstoff ausführliche Berechnungen der Leitfähigkeit $\sigma_{1}$ vor $^{5}$, nicht dagegen für $\sigma_{2}$ und $\sigma_{3}$. Wir wollen daher die Existenz der neutralen Atome in stark vereinfachter Weise durch ihren Einfluß auf die Stoßzeit $\tau_{\mathrm{e}}$ berücksichtigen:

$$
1 / \tau_{\mathrm{e}}=1 / \tau_{\mathrm{ei}}+1 / \tau_{\mathrm{e} 0} .
$$

$\tau_{\mathrm{e} 0}$ ist die mittlere Stoßzeit für Impulsaustausch zwischen Elektronen und Atomen:

$$
\tau_{\mathrm{e} 0}=\frac{3}{4} \cdot 1 /\left(v_{\mathrm{e}} n_{0} Q_{\mathrm{e} 0}\right)
$$

$\left(v_{\mathrm{e}}=\sqrt{8 k T / \pi m_{\mathrm{e}}}=\right.$ mittlere thermische Geschwindigkeit der Elektronen, $n_{0}=$ Dichte der Atome, $Q_{\mathrm{e} 0}$ $=$ Impulsaustauschquerschnitt).

Da die Berücksichtigung der Neutralteilchen nach Gl. (8) ohnehin nur eine sehr einfache Näherung darstellt, beschränken wir uns bei Gl. (9) auf das Stoßmodell harter, elastischer Kugeln mit $Q_{\mathrm{e} 0}=$ $20 \cdot 10^{-16} \mathrm{~cm}^{2} 5$. Da im schwach ionisierten Plasma die Stöße der Elektronen mit neutralen Atomen dominieren, deren Stoßquerschnitt wir konstant gesetzt haben, gilt dort $\sigma_{\|}=\sigma_{\perp}$. Aus Gründen der Einfachheit verwenden wir trotzdem weiterhin die $\mathrm{Gl}$. (3b). Dieser Fehler um etwa den Faktor 2 ist völlig vernachlässigbar gegen den starken Einfluß, den $\omega_{\mathrm{e}} \tau_{\mathrm{e}}$ im schwach ionisierten Gas auf das Verhältnis $\sigma_{2} / \sigma_{1}$ ausübt. Weiterhin bedeutet die Anwendung der Gl. (6), daß wir den Stromtransport durch $\nabla p_{0}$ vernachlässigen.

\section{Die Aufstellung der Differentialgleichung für das elektrische Potential}

\subsection{Spezialisierung des Ohmschen Gesetzes}

Den weiteren Rechnungen wird ein Zylinderkoordinatensystem $(r, \varphi, z)$ zugrunde gelegt. Die $z$ Richtung fällt mit der Symmetrieachse der experimentellen Anordnung zusammen und zeigt von der Anode zur Kathode.

5 R. S. Devoto, J. Plasma Phys. 2, 617 [1968].
Die spezielle Geometrie erlaubt Vereinfachungen des Ohmschen Gesetzes (6), die nun zusammen mit einigen Vernachlässigungen aufgeführt werden sollen:

a) Aus der Rotationssymmetrie $\partial / \partial \varphi=0$ und aus der Stationarität $\partial / \partial t=0$ folgt: $\boldsymbol{E}\left(E_{r}, 0, E_{z}\right)$.

b) Die Entladung brennt in einem homogenen, äußeren Magnetfeld $B_{0}(\geqq 1$ Tesla), das in $z$-Richtung zeigt. Die durch die ambipolare Diffusion und den Nernst-Effekt (Thermokraft proportional zu $\nabla \boldsymbol{T} \times \boldsymbol{B})$ verursachten diamagnetischen Ströme in $\varphi$-Richtung schwächen das axiale Magnetfeld ${ }^{6,7}$. Die Abnahme von $B_{z}$ vom Bogenrand zur Bogenachse liegt in der Größenordnung von $10^{-2}$ Tesla. Das durch den Strom in $z$-Richtung $\left(\approx 2 \cdot 10^{3} \mathrm{~A}\right)$ aufgebaute azimutale Magnetfeld $B_{\varphi}$ beträgt maximal etwa $4 \cdot 10^{-2}$ Tesla. Die durch diese Abweichungen von der Homogenität des Magnetfeldes verursachten Terme im Ohmschen Gesetz (hauptsächlich $j_{z} B_{\varphi}$ und $j_{r} B_{\varphi}$ ) werden vernachlässigt. Daher wird für $\mathbf{B}$ angesetzt: $\boldsymbol{B}\left(0,0, B_{0}\right)$.

c) Die radialen Druckdifferenzen im Plasma werden durch die diamagnetischen Ströme in $\varphi$-Richtung verursacht. Zusammen mit $B_{z}$ bewirken sie einen Anstieg des Druckes vom Bogenrand zur Bogenachse hin. Im zylindersymmetrischen Fall $(\partial / \partial z=0)$ läßt sich dieser Druckaufbau leicht berechnen ${ }^{7}$. Es ergibt sich, daß dann $p$ nur von der Temperatur abhängt. In einem Bogen endlicher Länge ist das nicht der Fall, da sich die durch das axiale Temperaturprofil verursachten Druckunterschiede längs des Magnetfeldes ausgleichen können. Außerdem wird sich der Druck weniger hoch aufbauen, wenn Gas axial aus dem Bogengefäß abströmen kann. Da sich bei den Experimenten, mit denen die Theorie später verglichen werden soll, der Druck auf den Elektroden und deren Halterung „abstützen“ kann, nehmen wir an, daß überall im Bogen das radiale Druckprofil herrscht, welches dem Querschnitt mit der höchsten Achsentemperatur entspricht. Aus diesen Überlegungen und aus der Rotationssymmetrie folgt: $\nabla p_{\mathrm{e}}\left(\partial p_{\mathrm{e}} / \partial r, 0,0\right)$. Bei bekannter Temperaturverteilung ist damit $p_{\mathrm{e}}$ durch die in ${ }^{7}$ berechnete Abhängigkeit vom Druck $p_{\mathrm{A}}$ außerhalb der heißen Zone $\left(T<6000^{\circ} \mathrm{K}\right)$, vom Magnetfeld $B_{0}$ und von der Temperatur gegeben. Im vollständig ionisierten Wasserstoff gilt: $p(T)=C_{\mathrm{e}} T^{3 / 4}$, wobei $C_{\mathrm{e}}$ von $p_{\mathrm{A}}$ und $B$ abhängt.

6 R. Wienecke, Z. Naturforsch. 18 a, 1151 [1963].

7 J. RAeder u. S. Wirtz, Z. Naturforsch. 23 a, 1695 [1968]. 
Unter Berücksichtigung der Punkte a) bis c) ergeben sich aus den Gln. (5) und (7) die Komponenten der elektrischen Stromdichte $\boldsymbol{j}$ zu:

$j_{r}=\sigma_{2} E_{r}+\frac{\sigma_{2}}{e n_{\mathrm{e}}} \frac{\partial p_{\mathrm{e}}}{\partial r}+B_{0}\left(\sigma_{2} v_{\varphi}-\sigma_{3} v_{r}\right)+\beta_{2} \frac{\partial T}{\partial r}$,

$j_{\varphi}=-\sigma_{3} E_{r}-\frac{\sigma_{3}}{e n_{\mathrm{e}}} \frac{\partial p_{\mathrm{e}}}{\partial r}-B_{0}\left(\sigma_{3} v_{\varphi}+\sigma_{2} v_{r}\right)-\beta_{3} \frac{\partial T}{\partial r}$,

$j_{z}=\sigma_{1} E_{z}+\beta_{1} \frac{\partial T}{\partial z}$.

\subsection{Die Differentialgleichung für das elektrische Potential}

Da alle betrachteten Vorgänge stationär sein sollen, verschwindet die Rotation des elektrischen Feldes und dieses kann aus einem skalaren Potential $\Phi$ berechnet werden.

$$
\boldsymbol{E}=-\nabla \Phi
$$

Aus der Bedingung

$$
\nabla \cdot \boldsymbol{j}=0
$$

der Rotationssymmetrie $\partial / \partial \varphi=0$ und den Gln. (10a), (10b), (10c) und (11) folgt für $\Phi$ in $\mathrm{Zy}$ linderkoordinaten die partielle Differentialgleichung:

$$
\frac{1}{r} \frac{\partial}{\partial r}\left(r \sigma_{2} \frac{\partial \Phi}{\partial r}\right)+\frac{\partial}{\partial z}\left(\sigma_{1} \frac{\partial \Phi}{\partial z}\right)=Q_{p}+Q_{v}+Q_{T}
$$

mit den Quelltermen

$$
\begin{gathered}
Q_{p}=\frac{1}{r} \frac{\partial}{\partial r}\left(r \frac{\sigma_{2}}{e n_{\mathrm{e}}} \frac{\partial p_{\mathrm{e}}}{\partial r}\right), \\
Q_{v}=\frac{1}{r} \frac{\partial}{\partial r}\left[r B_{0}\left(\sigma_{2} v_{\varphi}-\sigma_{3} v_{r}\right)\right], \\
Q_{T}=\frac{\partial}{\partial z}\left(\beta_{1} \frac{\partial T}{\partial z}\right)+\frac{1}{r} \frac{\partial}{\partial r}\left(r \beta_{2} \frac{\partial T}{\partial r}\right) .
\end{gathered}
$$

Für die Dichte der elektrischen Raumladung $\varrho_{\mathrm{el}}$ folgt aus dem Ansatz $\boldsymbol{D}=\varepsilon_{0} \boldsymbol{E}$ und

$$
\nabla \cdot \boldsymbol{D}=\varrho_{\mathrm{el}}
$$

mit den Gln. (13) und (14) :

$$
\begin{aligned}
\varrho_{\mathrm{el}}= & \frac{\varepsilon_{0}}{\sigma_{1}}\left[\left(\sigma_{2}-\sigma_{1}\right) \frac{1}{r} \frac{\partial}{\partial r}\left(r \frac{\partial \Phi}{\partial r}\right)\right. \\
& \left.+\frac{\partial \Phi}{\partial r} \frac{\partial \sigma_{2}}{\partial r}+\frac{\partial \Phi}{\partial z} \frac{\partial \sigma_{1}}{\partial z}-\left(Q_{p}+Q_{v}+Q_{T}\right)\right] .
\end{aligned}
$$

Die Gl. (16) zeigt die verschiedenen Ursachen elektrischer Raumladungen im Plasma, die das Vakuumfeld verzerren:

a) Die durch das Magnetfeld verursachte Anisotropie der elektrischen Leitfähigkeit (1. Summand).

b) Die Abhängigkeit der elektrischen Leitfähigkeit vom Ort, die z. B. durch eine Temperaturverteilung verursacht werden kann (2. und 3 . Summand).

c) Die stromtreibenden Kräfte, die nicht durch den Gradient eines skalaren elektrischen Potentials beschrieben werden können $\left(Q_{p}, Q_{v}, Q_{T}\right)$.

\section{Analytische Lösungen der Potentialgleichung}

Um zunächst grundsätzlich den Einfluß des Magnetfeldes auf das elektrostatische Potential im Lichtbogen studieren zu können, soll die Gl. (13) durch folgende Annahmen vereinfacht werden:

a) Die Leitfähigkeiten $\sigma_{1}$ und $\sigma_{2}$ nehmen konstante Werte an, die aber voneinander verschieden sein können.

b) Alle stromtreibenden Kräfte außer dem elektrischen Feld im Laborsystem wurden vernachlässigt, d. h. alle Quellterme $Q$ verschwinden.

Diesen Annahmen entspricht ein räumlich homogenes Plasma ohne Schwerpunktströmung quer zum Magnetfeld. Die Gl. (13) lautet dann:

$$
\frac{1}{r} \frac{\partial}{\partial r}\left(r \frac{\partial \Phi}{\partial r}\right)+\frac{\sigma_{1}}{\sigma_{2}} \frac{\partial^{2} \Phi}{\partial z^{2}}=0 .
$$

\subsection{Punktförmige Quellen und Senken des elektrischen Stromes}

Mit der Substitution

$$
w=\sqrt{\sigma_{2} / \sigma_{1}} z=\alpha z
$$

geht die Gl. (17) in die Laplacesche Differentialgleichung über:

$$
\frac{1}{r} \frac{\partial}{\partial r}\left(r \frac{\partial \Phi}{\partial r}\right)+\frac{\partial^{2} \Phi}{\partial w^{2}}=0 .
$$

Wenn man die Elektroden des Bogens zu Punkten idealisiert und als Randbedingung das Verschwinden des Potentials im Unendlichen fordert, so kann man die Lösung der Gl. (19) leicht angeben. Sie entsteht durch Superposition von zwei Grundlösungen. Bei Annahme einer Quelle (Anode) bei $r=0$, $z=0$ und der entsprechenden Senke (Kathode) bei $r=0, z=L$ erhält man $\Phi(r, w)=A\left\{\left[r^{2}+w^{2}\right]^{-1 / 2}-\left[r^{2}+(w-\alpha L)^{2}\right]^{-1 / 2}\right\}$. 
Macht man die Substitution (18) wieder rückgängig und drückt die Konstante $A$ durch den aus der Quelle fließenden Strom $I$ aus, so erhält man die Potentialverteilung:

$$
\begin{gathered}
\Phi(r, z)=\left(I / 4 \pi \sqrt{\sigma_{1} \sigma_{2}}\right)\left\{\left[r^{2}+\left(\sigma_{2} / \sigma_{1}\right) z^{2}\right]^{-1 / 2}\right. \\
\left.-\left[r^{2}+\left(\sigma_{2} / \sigma_{1}\right)(z-L)^{2}\right]^{-1 / 2}\right\} .
\end{gathered}
$$

Am deutlichsten sichtbar wird der Einfluß des Magnetfeldes - ausgedrückt durch das Verhältnis $\sigma_{2} / \sigma_{1}$ - bei Betrachtung des radialen Profils der Feldstärke $E_{z}$ in der Mittelebene $z=L / 2$. Die auf den Wert in der Achse normierte Verteilung von $E_{z}$ erhält man aus den Gln. (11) und (20) zu:

$$
\frac{E_{z}\left(\varrho^{*}, L / 2\right)}{E_{z}(0, L / 2)}=\frac{1}{\left(1+\varrho^{* 2}\right)^{3 / 2}}
$$

mit

$$
\varrho^{*}=\sqrt{\sigma_{1} / \sigma_{2}} \cdot 2 r / L \text {. }
$$

Aus der graphischen Darstellung der Gl. (21) (Abb. 2) ersieht man, daß die normierte Feldstärke bei $\varrho_{j}^{*}=2$ etwa auf den Wert $1 / 10$ abgefallen ist.

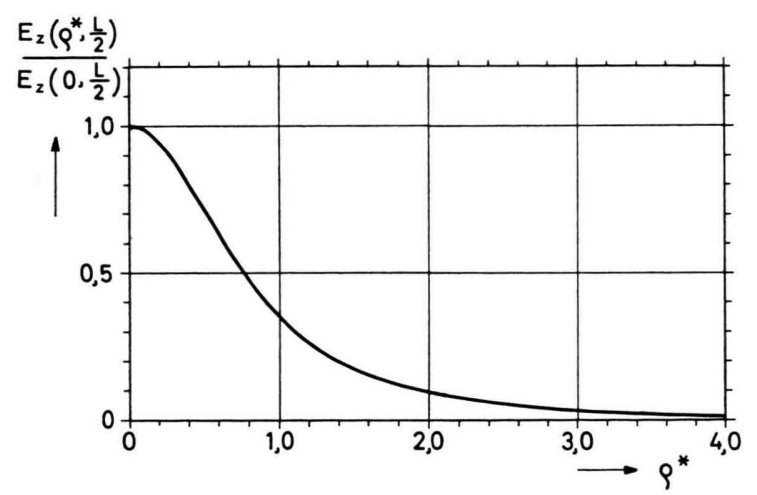

Abb. 2. $E_{z}$ bei $z=L / 2$ als Funktion des normierten Radius $\varrho^{*}$.

Dieser Radius wird als Maß für die Breite der Feldstärkeverteilung gewählt. Für den maximalen Radius $r_{j}$ des Profils von $E_{z}$ zwischen Quelle und Senke folgt daraus:

$$
r_{j}=\sqrt{\sigma_{2} / \sigma_{1}} \cdot L
$$

Für $\omega_{\mathrm{e}} \tau_{\mathrm{e}} \gg 1$ geht Gl. (23) über in:

$$
r_{j}=L / \omega_{\mathrm{e}} \tau_{\mathrm{e}} .
$$

Die Formel (23) zeigt nun sehr deutlich den Einfluß des Magnetfeldes auf die Feldstärke- und Stromdichteverteilung. Für $B=0$ gilt $\sigma_{2} / \sigma_{1}=1$, d. h. $r_{j}=L$. Die Breite der Stromdichteverteilung wird dann ebenso groß wie der Elektrodenabstand. Für große $B$ gilt $\sigma_{2} / \sigma_{1}=1 /\left(\omega_{\mathrm{e}} \tau_{\mathrm{e}}\right)^{2}$, d. h. $r_{j}=L / \omega_{\mathrm{e}} \tau_{\mathrm{e}}$. Ein starkes Magnetfeld reduziert also die radiale Ausdehnung des Stromdichteprofils um den Faktor $1 / \omega_{\mathrm{e}} \tau_{\mathrm{e}}$ und führt damit zu einer Konzentration des Stromes um die magnetische Feldlinie, welche die punktförmigen Elektroden verbindet.

Um ein anschauliches Bild von der Stromverteilung zu gewinnen, sollen nun noch die Stromlinien von $\boldsymbol{j}$ in der $r, z$-Ebene berechnet werden. Da $\boldsymbol{j}$ wegen des Hall-Effektes auch eine $\varphi$-Komponente besitzt [Gl. (10b)], winden sich diese Stromlinien $r(z)$ spiralförmig um die $z$-Achse. Die Differentialgleichung der Stromlinien $r(z)$ lautet:

$$
\mathrm{d} r / \mathrm{d} z=j_{r} / j_{z} .
$$

Daraus folgt mit den Gln. (10a), (10c), (11) und (20) :

$$
\frac{\mathrm{d} r}{\mathrm{~d} z}=\frac{2 r\left(N_{1}-N_{2}\right)}{2 z\left(N_{1}-N_{2}\right)-\left(N_{1}+N_{2}\right)}
$$

mit

$$
\begin{aligned}
& N_{1}=\left[r^{2}+\alpha^{2} z^{2}\right]^{3 / 2}, \\
& N_{2}=\left[r^{2}+\alpha^{2}(z-L)^{2}\right]^{3 / 2} .
\end{aligned}
$$

Die Differentialgleichung (25) hat singuläre Punkte bei $r=0, z=0$ und $r=0, z=L$, durch die alle Lösungskurven laufen müssen. Dieses Verhalten entspricht natürlich der Konstruktion der Potentialverteilung aus zwei Grundlösungen. Als Scharparameter der Lösungskurven kann man z. B. den Winkel gegen die $z$-Achse verwenden, unter dem die Stromlinien aus der Quelle austreten. Wir parametrisieren so, daß der gegenseitige Abstand der Stromlinien ein $\mathrm{Ma} \beta$ für die Stromdichte ist. In Abb. 3 ist ein mit Gl. (25) numerisch berechnetes Stromlinienbild

$$
\sigma_{2} / \sigma_{1}=10^{-2}
$$

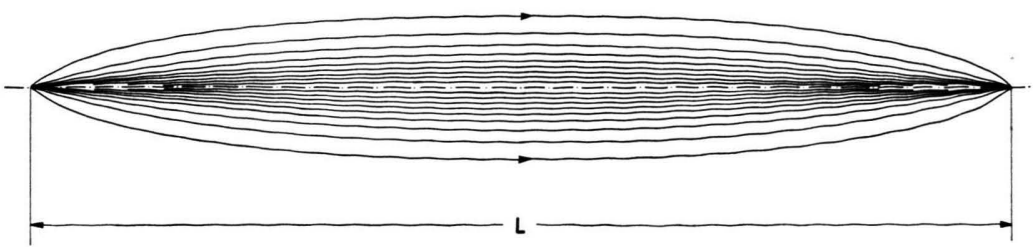

Abb. 3. Bild der Stromlinien zwischen punktförmiger Quelle und Senke. 
für $\alpha=10^{-1}$ aufgezeichnet. Bilder für andere Werte von $\alpha$ erhält man einfach durch Verzerrung der $r$ Koordinate im Verhältnis der verschiedenen $\alpha$.

\subsection{Ebene, kreisförmige, koaxiale Elektroden}

Einen Schritt weiter hin zur experimentellen Realität führt der Ersatz punktförmiger Elektroden durch ebene Elektroden endlicher Größe. Die Potentialverteilung in einer solchen Anordnung kann man sich als Integral über Quellströmungen der bisher berechneten Art vorstellen. Diese vermitteln daher schon eine qualitative Vorstellung über das Feld zwischen ausgedehnten Elektroden, das danach gemäß Gl. (23) etwa im Abstand $L \sqrt{\sigma_{2} / \sigma_{1}}$ vom äußeren Elektrodenrand abgeklungen sein wird. Für den Fall ebener, kreisförmiger Elektroden gleicher Größe ist die Lösung der Gl. (19) bekannt. Formal entspricht sie der Berechnung des Feldes in einem Plattenkondensator, dessen Plattenabstand um den Faktor $a$ verkleinert ist. Mit den Bezeichnungen der

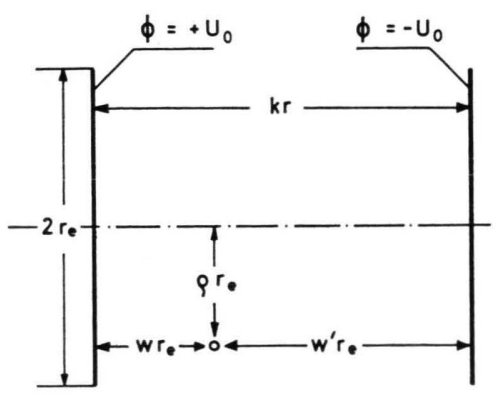

Abb. 4. Zur Berechnung des Potentials zwischen zwei ebenen kreisförmigen Platten. Der axiale Plattenabstand beträgt $k r_{\mathrm{e}}$, nicht $k r$ wie irrtümlich in der Abb. angegeben.

Abb. 4 besteht die Lösung aus den beiden Gleichungen $^{8}$ :

$$
\begin{gathered}
\Phi\left(\varrho, w, w^{\prime}\right)=\frac{U_{0}}{\pi} \int_{-1}^{1}\left\{\frac{1}{\sqrt{\varrho^{2}+(w+i t)^{2}}}\right. \\
\left.-\frac{1}{\sqrt{\varrho^{2}+\left(w^{\prime}+i t\right)^{2}}}\right\} f(t) \mathrm{d} t, \\
f(t)=1+\frac{1}{\pi} \int_{-1}^{1} \frac{k}{k^{2}+(t-y)^{2}} f(y) \mathrm{d} y .
\end{gathered}
$$

Dabei wurden folgende Randbedingungen verwendet:

8 I. N. Sneddon, Mixed Boundary Value Problems in Potential Theory, North-Holland Publ. Co., Amsterdam, und J. Wiley \& Sons, Inc., New York 1966. a) Die Elektroden besitzen entgegengesetzt gleiches Potential $\left(+U_{0}\right.$ bzw. $\left.-U_{0}\right)$.

b) Im Unendlichen verschwindet das Potential.

Die Funktion $f(t)$ ist reell, gerade und wird durch die Fredholmsche Integralgleichung zweiter Art (26b) bestimmt. Deren Lösung kann man durch die Neumannsche Reihe darstellen. Wenn $f(t)$ bekannt ist, kann man das Potential $\Phi$ nach Gl. (26a) durch Integration berechnen. Die Komponenten des elektrischen Feldes werden durch formale Differentiation unter dem Integralzeichen nach den Koordinaten aus Gl. (26a) berechnet. Bei allen vorkommenden Wurzeln muß immer der positive Realteil verwendet werden.

Wir haben die Gl. (26b) ausgehend von einer einfachen Näherung für $f(t)$ iterativ gelöst. Ein Vergleich mit Rechnungen von Fox und Goodwin ${ }^{9}$, die ein anderes Verfahren verwenden, für den Fall $k=1$ ergibt vollständige Übereinstimmung. Die Abb. 5 zeigt als Ergebnis der numerischen Lösung der Gln. (26a) und (26b) die Verteilung der axialen elektrischen Feldstärke $E_{z}$ in Abhängigkeit von der normierten radialen Koordinate $r / r_{\mathrm{e}}$. Die Verteilungen gelten für die Mitte zwischen Elektroden, die sich im Abstand $L / r_{\mathrm{e}}=100$ gegenüberstehen. Um einen klaren Vergleich der Kurvenform zu ermöglichen, sind die Feldstärken auf den jeweiligen Achsenwert normiert. Den Parameterwerten $\sigma_{2} / \sigma_{1}$ $=1,10^{-2}$ und $10^{-4}$ entsprechen etwa die Werte $\omega_{\mathrm{e}} \tau_{\mathrm{e}}=0,10$ und 100 (s. Anhang). Die Abb. 5 zeigt sehr deutlich, daß das elektrische Vakuumfeld durch das magnetisierte Plasma drastisch im Sinne einer zunehmenden Konzentration auf die durch die Elektrodenradien bestimmten magnetischen Flußröhre verändert wird.

Die physikalische Anschauung legt die Vermutung nahe, daß $E_{z}$ nach Abb. 5 um die Strecke $r_{j} / r_{\mathrm{e}}=\left(L / r_{\mathrm{e}}\right) \sqrt{\sigma_{2} / \sigma_{1}}$ [Gl. (23)] über den Radius $r / r_{\mathrm{e}}=1$ hinausgreift. Für den maximalen, auf $r_{\mathrm{e}}$ normierten Radius der Flußröhren von $E_{z}$ und $j_{z}$ erhält man damit einfach:

$$
r / r_{\mathrm{e}}=1+\sqrt{\sigma_{2} / \sigma_{1}} \cdot L / r_{\mathrm{e}} .
$$

Da der Effekt der Stromkonzentration nur für $\omega_{\mathrm{e}} \tau_{\mathrm{e}} \gg 1$ interessant wird, kann man die Gl. (27) noch zu

$$
r / r_{\mathrm{e}}=1+\left(1 / \omega_{\mathrm{e}} \tau_{\mathrm{e}}\right) \cdot L / r_{\mathrm{e}}
$$

${ }^{9}$ L. Fox u. E. T. Goodwin, Phil. Trans. Roy. Soc. A 245, 501 [1953]. 


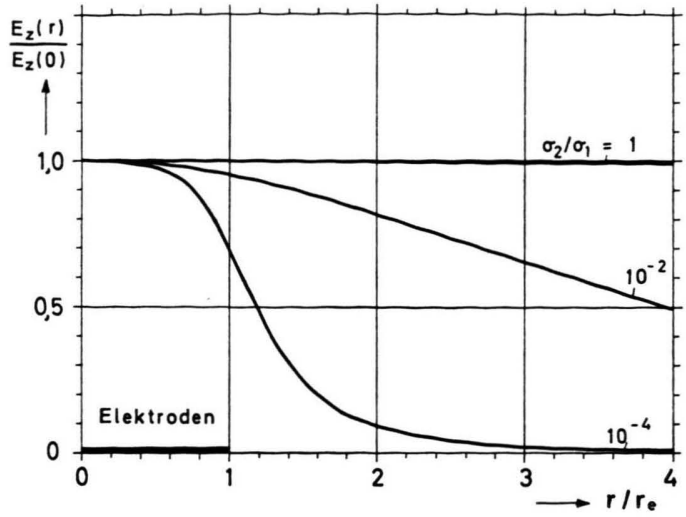

Abb. 5. Radiale Profile von $E_{z}$ bei $z=L / 2$ für verschiedene Werte von $\sigma_{2} / \sigma_{1}$.

vereinfachen. Um die Ableitung der Gln. (27) bzw. (27a) nicht nur plausibel zu begründen, wurden die Radien, bei denen $E_{z}$ und damit $j_{z}$ auf $1 / 10$ des Achsenwertes abgefallen sind, für die Mitte zwischen den Elektroden aus den Gln. (26a) und (26b) berechnet. Die Ergebnisse sind in Abb. 6 aufgezeich-

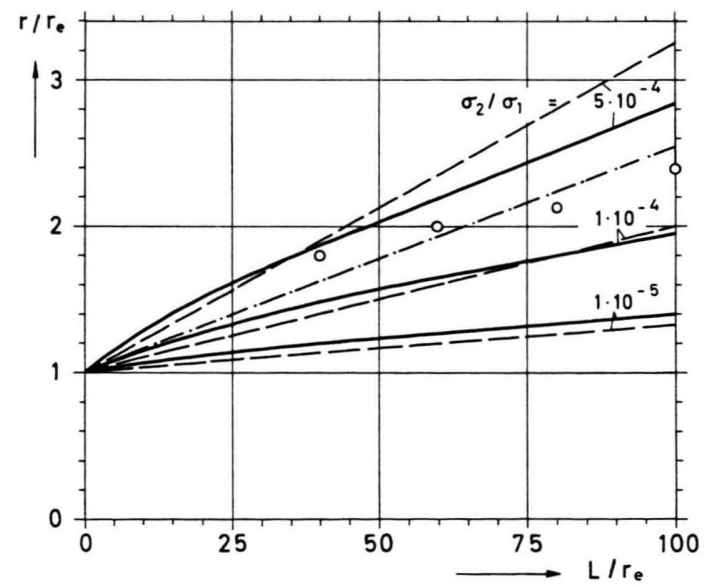

Abb. 6. Radien des $E_{z}$-Profils bei $z=L / 2$. Durchgezogene Kurve: exakte Werte, gestrichelte Gerade: Näherung nach Gl. (27), Kreise: experimentelle Werte nach ZANKL ${ }^{2}$, strichpunktierte Gerade: Approximation der experimentellen Werte nach Gl. (27).

net. Zum Vergleich wurden auch die durch die Gl. (27) gegebenen Geraden gestrichelt eingetragen. Sie zeigen, daß die einfachen Formeln (27) bzw. (27a) für große Werte von $\omega_{\mathrm{e}} \tau_{\mathrm{e}}$ zumindest für Abschätzungen gut geeignet sind. ZANKL hat die Abhängigkeit des Bogenradius vom Elektrodenabstand an einem Wasserstoffbogen untersucht ${ }^{2}$. Seine Ergebnisse für ein Magnetfeld $B_{0}=40 \mathrm{kG}$ und einen Kathodenradius von $7,5 \mathrm{~mm}$ sind ebenfalls in Abb. 6 eingezeichnet. Bei einem Vergleich mit der Theorie muß allerdings beachtet werden, daß der Radius bei diesen Experimenten durch den Abfall der Temperatur auf $10^{4}{ }^{\circ} \mathrm{K}$ definiert wurde. Der Zusammenhang zu unserer Definition wird durch die Tatsache hergestellt, daß für Temperaturen unterhalb von $10^{4}{ }^{\circ} \mathrm{K}$ der Hall-Parameter $\omega_{\mathrm{e}} \tau_{\mathrm{e}}$ sehr stark mit fallender Temperatur ansteigt (Abb. $12 \mathrm{a}$ ). Die Feldstärke $E_{z}$ klingt in diesem Bereich deshalb stark mit zunehmendem Radius ab. Paßt man die Gl. (27a) an die Meßwerte an, so erhält man: $\omega_{\mathrm{e}} \tau_{\mathrm{e}} \approx 62$. Der Mechanismus zur Stabilisierung des Bogens wirkt also besonders in dem radialen Abstandsbereich, in dem noch hohe Werte von $\omega_{\mathrm{e}} \tau_{\mathrm{e}}$ vorliegen.

\section{Numerische Berechnung der Potential- verteilung}

Die bisherigen Rechnungen, denen physikalisch die Annahme eines homogenen Plasmas entspricht, konnten nur dazu dienen, die Parameter klar herauszuarbeiten, von denen die Potentialverteilung im magnetisierten Plasma endlicher Länge abhängt. Bei einer quantitativen Untersuchung muß berücksichtigt werden, daß die Leitfähigkeitskoeffizienten $\sigma$ von der Temperatur und vom Elektronendruck abhängen. Außerdem beeinflussen nach den Gln. (14a), (14b), (14c) die Schwerpunktsgeschwindigkeit $v$ und die Gradienten von Druck und Temperatur die Potentialverteilung. Die Koeffizienten der Differentialgleichung (13) hängen bei Annahme einer realistischen Temperaturverteilung so komplizert vom Ort $a b$, daß nur eine numerische Lösung Erfolg verspricht. Zunächst soll die - zur Vermeidung unnötiger rechnerischer Komplikationen - idealisierte Geometrie zusammen mit den Randbedingungen angegeben werden (Abb. 7).

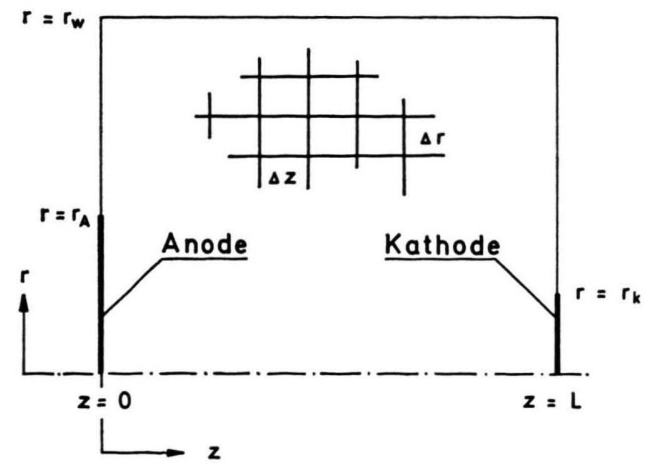

Abb. 7. Zur numerischen Lösung der Potentialgleichung. 


\subsection{Geometrische Anordnung und Randbedingungen}

Die Elektroden werden zu ebenen Kreisscheiben idealisiert, die wie im Experiment verschiedene Durchmesser besitzen können. Die Anordnung wird von einem isolierenden Zylinder mit dem Radius $r_{\mathrm{w}}$ umschlossen. Außerdem wird angenommen, daß durch die Kreisringe zwischen den Elektroden und der Zylinderwand kein Strom fließen kann. Die Randbedingungen für die Gl. (13) lauten:

$$
\begin{aligned}
& \frac{\partial \Phi}{\partial r}=0 \quad \text { bei } \quad r=0, \quad 0 \leqq z \leqq L \text {; } \\
& j_{z}=0 \quad \text { bei } \quad r_{\mathrm{A}}<r \leqq r_{\mathrm{w}}, \quad z=0 ; \\
& j_{z}=0 \quad \text { bei } \quad r_{\mathrm{K}}<r \leqq r_{\mathrm{w}}, \quad z=L \text {; } \\
& \text { (28c) } \\
& j_{r}=0 \quad \text { bei } \quad r=r_{\mathrm{w}}, \quad 0<z<L \text {; } \\
& \text { (28d) } \\
& \left.\begin{array}{l}
2 \pi \int_{0}^{r_{\mathrm{A}}} j_{z} r \mathrm{~d} r=I \\
\Phi=\mathrm{const}
\end{array}\right\} \text { bei } \quad 0 \leqq r \leqq r_{\mathrm{A}}, \quad z=\underset{(28 \mathrm{e})}{0} \\
& \Phi=\Phi_{\mathrm{K}} \quad \text { bei } \quad 0 \leqq r \leqq r_{\mathrm{K}}, \quad z=L \text {. }
\end{aligned}
$$

Die Bedingung (28a) folgt aus der Rotationssymmetrie. Die Gln. (28e) sind nicht unabhängig voneinander, sondern müssen simultan erfüllt werden. Aus der Forderung, da $\beta$ der gesamte Strom $I$ durch die Anode fließen soll und der Tatsache, daß die Anode eine Äquipotentialfläche darstellt, ergibt sich das Anodenpotential $\Phi_{\mathrm{A}}$. Der Wert $\Phi_{\mathrm{A}}$ kann daher bei gegebenem Strom $I$ nicht willkürlich gewählt werden. Die Bedeutung der übrigen Randbedingungen ist offensichtlich. Die in der Abb. 7 dargestellte Anordnung wird mit einem Gitter der Maschenweiten $\Delta z$ und $\Delta r$ überzogen, in dessen Knotenpunkten die Werte von $\Phi$ numerisch berechnet werden sollen.

\subsection{Aufstellung der Differenzengleichungen}

Da die Transportkoeffizienten im Ohmschen Gesetz von der Temperatur und vom Elektronendruck abhängen, die Potentialgleichung (13) dagegen nur eine der Gleichungen aus einem geschlossenen System von Plasmagleichungen darstellt, muß die räumliche Verteilung von $T$ vorgegeben werden. Die Verteilung von $p_{\mathrm{e}}$ ist dann durch die Annahme c) in
Abschnitt 2 bestimmt. Durch diese Entkopplung der Potentialgleichung von der Energiebilanz und der Kraftgleichung entsteht aus der Gl. (13) eine lineare, elliptische Differentialgleichung mit ortsabhängigen Koeffizienten.

Zur numerischen Berechnung der Potentialverteilung muß nun ein lineares Gleichungssystem aufgestellt werden, das die Werte von $\Phi$ an allen Gitterpunkten bestimmt. Will man dabei von der Differentialgleichung für $\Phi$ ausgehen, so ist es günstig, direkt die in Gl. (13) vorliegenden Differentialoperatoren durch zentrale Differenzenformeln zu approximieren, d. h. die Ausdrücke in Klammern nicht auszudifferenzieren. Das so entstehende Gleichungssystem erfüllt dann den Erhaltungssatz $\nabla \cdot \boldsymbol{j}=0$ in jedem Volumelement des diskreten Schemas. Wegen dieser Eigenschaft kann man das Gleichungssystem auch direkt aus der Divergenzfreiheit der Stromdichte $\boldsymbol{j}$ ableiten ${ }^{10}$. Dazu integrieren wir die Bedingung $\nabla \cdot \boldsymbol{j}=0$ über ein Volumelement unseres Gitters (Abb. 8) und formen das Integral mit Hilfe

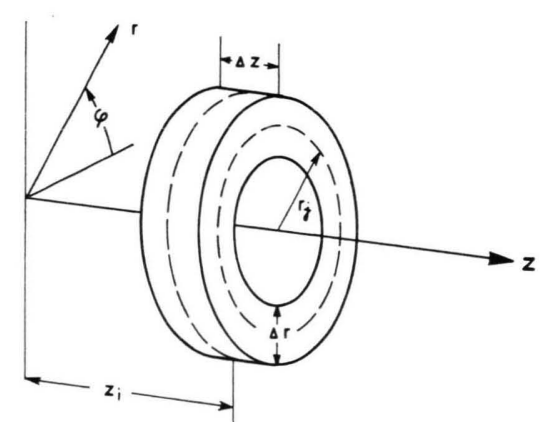

Abb. 8. Volumelement bei der numerischen Lösung der Potentialgleichung.

des Gaußschen Satzes in das Oberflächenintegral der Normalkomponenten von $\boldsymbol{j}$ um. Die Komponenten $j_{r}$ und $j_{z}$ sind durch die Gln. (10a) und (10c) gegeben. Die ortsabhängigen Größen $\sigma, k$ und $n_{\mathrm{e}}$ werden bei der Integration durch ihre Werte in der Mitte der entsprechenden Oberflächenelemente, die Ableitungen von $T$ und $p_{\mathrm{e}}$ durch zentrale Differenzenformeln von der Ordnung $(\Delta z)^{2}$ bzw. $(\Delta r)^{2}$ approximiert. Zusammen mit den Randbedingungen $(28 \mathrm{a}-\mathrm{f})$ entsteht so ein lineares Gleichungssystem, das ein diskretes mathematisches Modell für die differentielle Bedingung $\nabla \cdot \boldsymbol{j}=0$ darstellt.

10 E. L. Wachspress, Iterative Solution of Elliptic Systems, Prentice-Hall, Inc., Englewood Cliffs 1966. 


\subsection{Lösung des Gleichungssystems}

Dieses Gleichungssystem wird iterativ nach dem Verfahren der sukzessiven Überrelaxation gelöst ${ }^{\mathbf{1 1}}$. Bevor die Resultate für verschiedene Spezialfälle diskutiert werden, sollen noch einige typische Parameter der numerischen Rechnung angegeben werden:

a) In $z$-Richtung betrug die Anzahl der Gitterpunkte $20-35$, in $r$-Richtung $40-60$, wobei auf den Radius der Kathode 15 - 20 Intervalle $\Delta r$ ententfielen. Diesen Unterteilungen entsprechen Systeme, die aus etwa $800-2100$ Gleichungen bestehen.

b) Der optimale Relaxationsfaktor $\omega$ hängt von den Koeffizienten des Gleichungssystems und damit von den verschiedenen Modellen ab. Da die Erfahrung jedoch gezeigt hatte, daß diese Abhängigkeit nicht stark ist, wurde im allgemeinen der für einen typischen Fall optimierte Wert $\omega=1,9$ verwendet.

c) Eine bequeme Kontrolle der Konvergenz besteht in der Integration der Stromdichte $j_{z}$ über Querschnitte bei verschiedenen Werten von $z$. Bei allen angegebenen Resultaten war der relative Fehler in allen Querschnitten kleiner als $10^{-2}$. Dieses Ergebnis wurde, je nach der Zahl der Gleichungen und der Ortsabhängigkeit der Koeffizienten, nach 100 bis 1000 Iterationen erreicht. Die dazu benötigte Rechenzeit auf der Rechenanlage IBM 360/91 betrug 10-60 Sekunden.

\subsection{Ergebnisse für verschiedene Spezialfälle}

\subsubsection{Homogenes Plasma}

Zunächst wurde die Potentialverteilung in einem homogenen Plasma ohne Strömung (s. Abschn. 3.2) für die Randbedingungen $(28 \mathrm{a}-\mathrm{f})$ berechnet. Dabei wurden $\Phi_{\mathrm{A}}=1, \Phi_{\mathrm{K}}=-1$ gesetzt, da die Lösung wegen $Q=0$ mit einem beliebigen Faktor multipliziert werden darf. Der Strom $I$ war dadurch vollständig bestimmt. Das Ergebnis für $\sigma_{2} / \sigma_{1}=10^{-4}$, $r_{\mathrm{K}}=r_{\mathrm{A}}, L / r_{\mathrm{K}}=100, r_{\mathrm{W}} / r_{\mathrm{K}}=4$ zeigt die Abb. 9 . In ein durch die analytische Lösung nach Abschn. 3.2 gegebenes Potentialgebirge sind numerische Ergebnisse eingetragen. In Achsennähe stimmen die Lösungen überein, weiter außen fallen die numerischen Ergebnisse stärker mit $r$ ab als die analytischen. Der Grund liegt in den verschiedenen Rand-

11 R. S. VArga, Matrix Iterative Analysis, Prentice-Hall, Inc., Englewood Cliffs 1962.

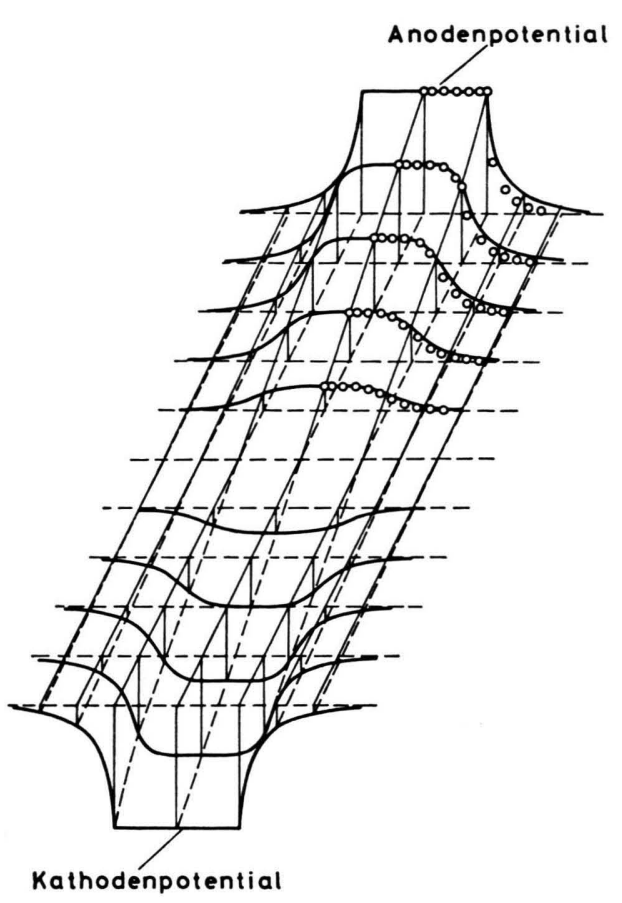

Abb. 9. Potentialgebirge zwischen zwei ebenen kreisförmigen Platten. Kreise: Ergebnise der numerischen Lösung.

bedingungen. Bei der analytischen Lösung verschwindet $\Phi$ im Unendlichen, während die numerische Lösung $j_{\mathrm{n}}=0 \quad\left(j_{\mathrm{n}}=\right.$ Normalkomponente der Stromdichte) und damit $\partial \Phi / \partial n=0$ auf allen Rändern außerhalb der Elektroden fordert. Die Unterschiede sind aber nicht sehr groß. Die numerische Lösung bestätigt damit die Möglichkeit, die in Abschn. 3.2 abgeleiteten Resultate auch auf ein durch endliche Ränder eingeschlossenes Plasma anzuwenden.

\subsubsection{Der Einfluß der axialen Temperatur- verteilung}

Da wir die Temperaturverteilung als gegebene Ortsfunktion betrachten, ist es wichtig, deren Einfluß zu studieren. Die radialen Temperaturprofile in Wasserstoffbögen im Magnetfeld sind gut bekannt 1, 2,12 und lassen sich für diese Modellrechnungen genügend gut durch eine Glockenkurve darstellen. Den Einfluß der axialen Verteilungen untersuchen wir durch die Darstellung des Temperaturprofils durch Parabeln verschiedener Ordnung.

12 D. Ludwig, Bericht des Instituts für Plasmaphysik, IPP $3 / 88$. 
Für $T(r, z)$ verwenden wir die Formel:

$$
\begin{aligned}
T(r, z)= & \left(T_{\max }-T_{\mathrm{w}}\right) \frac{\exp \left(-r^{2} / b^{2}\right)-\exp \left(-r_{\mathrm{w}}^{2} / b^{2}\right)}{1-\exp \left(-r_{\mathrm{w}}^{2} / b^{2}\right)} \\
& \cdot\left[1-\frac{T_{\max }-T_{\mathrm{e}}}{T_{\max }-T_{\mathrm{w}}} 2^{n}\left(\frac{z}{2}-\frac{1}{2}\right)^{n}\right]+T_{\mathrm{w}}, \quad n=2,4,6, \ldots .
\end{aligned}
$$

$T_{\max }$ ist die Temperatur bei $z=L / 2$ und $r=0, T_{\mathrm{e}}$ ist die Temperatur bei $r=0$ vor den Elektroden, $b$ bestimmt die radiale Ausdehnung des Temperaturprofils, die Zahl $n$ den axialen Verlauf. Die Kurven $1-3$ auf Abb. 10 zeigen den Verlauf des Potentials und der Temperatur auf der Bogenachse für

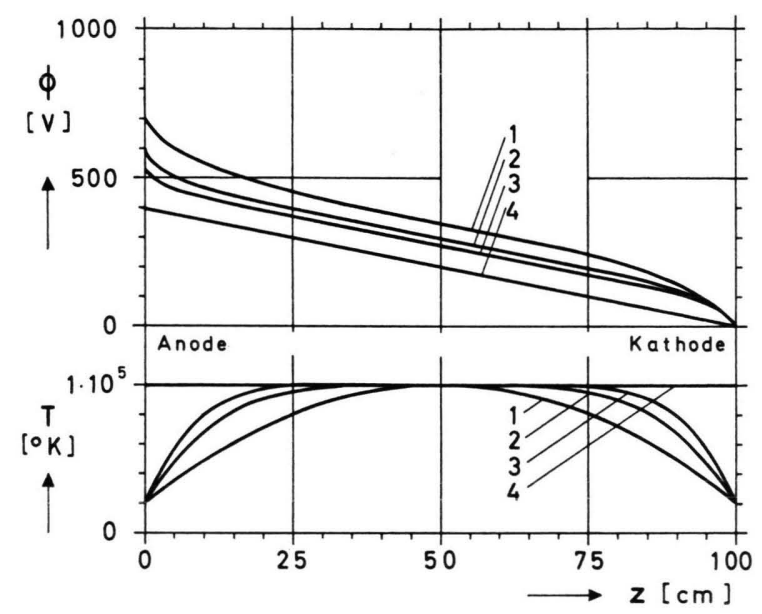

Abb. 10. Verschiedene axiale Temperaturverteilungen mit den entsprechenden axialen Potentialverteilungen bei $r=0$.

folgende Parameterwerte: $r_{\mathrm{K}}=r_{\mathrm{A}}=1 \mathrm{~cm}, r_{\mathrm{W}}=3 \mathrm{~cm}$, $\mathrm{L}=100 \mathrm{~cm}, T_{\max }=1 \cdot 10^{5}{ }^{\circ} \mathrm{K}, T_{\mathrm{e}}=2 \cdot 10^{4}{ }^{\circ} \mathrm{K}, T_{\mathrm{W}}$ $=10^{3}{ }^{\circ} \mathrm{K}, p_{\mathrm{A}}=10^{3} \mathrm{~N} / \mathrm{cm}^{2}, B_{0}=5$ Tesla, $I=2 \cdot 10^{3} \mathrm{~A}$, $b=1 \mathrm{~cm}, n=2,4$ und 6 . In der Umgebung von $z=L / 2$ laufen alle Potentialkurven parallel und ergeben daher die gleiche Feldstärke $E_{z}$. Dieses Resultat ergab sich auch außerhalb der Achse. Außerdem wurde noch der Fall $T_{\mathrm{e}}=T_{\max }$ berechnet (Kurve 4 ), bei dem $E_{z}$ praktisch längs des gesamten Bogens konstant ist. Auch hier ist die Verteilung von $E_{z}$ bei $z=L / 2$ fast identisch mit den Fällen $n=2,4$ und 6 . Wenn man nicht am genauen Verlauf von $E_{z}$ an den Enden des Bogens interessiert ist, kann man also die axiale Variation von $T$ vernachlässigen, zumal alle vorliegenden theoretischen und experimentellen Untersuchungen wegen der hohen axialen Wärmeleitfähigkeit ein flaches Temperaturprofil in Bogenmitte ergeben. Unmittelbar vor den Elektroden wird $E_{z}$ wegen der niedrigeren Temperatur immer stark ansteigen. Diese Bereiche werden aber ohnehin durch die Elektrodenmechanismen beherrscht, die wir bei unseren Rechnungen völlig vernachlässigt haben. Die Temperatur $T_{\mathrm{e}}$ soll daher als die Gastemperatur unmittelbar vor den Elektroden verstanden werden. Zur Berechnung der Spannungen zwischen den Elektroden müßte man deshalb die Elektrodenfälle zu den Anodenpotentialen nach Abb. 10 addieren.

Die Symmetrie der Kurven 1-3 auf Abb. 10 zeigt, daß bei den heute in Wasserstoffbögen experimentell erreichten Temperaturen von $1-2 \cdot 10^{5}{ }^{\circ} \mathrm{K}$ noch keine sehr wesentliche Stromdichte durch die axialen Temperaturgradienten verursacht wird. Da der Koeffizient $\beta_{1}$ jedoch mit $T$ zunimmt, steigt mit $T$ auch der Anteil der Stromdichte $\beta_{1} \cdot \partial T / \partial z$ relativ zur gesamten Stromdichte. Dieser Einfluß soll an einem Temperaturprofil mit hoher Maximaltemperatur $T_{\max }$ und starken axialen Gradienten der Temperatur demonstriert werden: $r_{\mathrm{K}}=r_{\mathrm{A}}=1 \mathrm{~cm}, r_{\mathrm{W}}$ $=3 \mathrm{~cm}, L=100 \mathrm{~cm}, T_{\max }=5 \cdot 10^{5}{ }^{\circ} \mathrm{K}, T_{\mathrm{e}}=10^{5}{ }^{\circ} \mathrm{K}$, $T_{\mathrm{W}}=10^{3}{ }^{\circ} \mathrm{K}, \quad p_{\mathrm{A}}=5 \cdot 10^{2} \mathrm{~N} / \mathrm{m}^{2}, \quad B=5$ Tesla, $I=$ $5 \cdot 10^{3} \mathrm{~A}$. Der hohe Wert für $T_{\mathrm{e}}$ wurde zur Vermeidung numerischer Schwierigkeiten gewählt. Einerseits reduziert er die Größe von $\partial T / \partial z$ nicht wesentlich, erlaubt aber andererseits noch eine nicht $\mathrm{zu}$ kleine axiale Schrittweite. Würde man z. B. $T_{\mathrm{e}}=10^{3}$ wählen, so würde diese Annahme sehr große Potentialgradienten vor den Elektroden und damit ein sehr feines Gitter erzwingen, ohne daß damit eine wesentliche Information gewonnen würde.

Die Abb. 11 zeigt die Potentialverläufe bei $r=0$ ohne (Kurve 1) und mit (Kurve 2) Berücksichtigung von $\nabla_{\|} T$ im Ohmschen Gesetz. Die Kurve 2 zeigt die Temperaturabhängigkeit des Effektes sehr deutlich. Unmittelbar vor der Anode ist $\nabla_{\|} T$ zwar schon groß, doch der Wert von $\beta_{1}$ bei $10^{5}{ }^{\circ} \mathrm{K}$ ist noch nicht hoch genug, um einen nennenswerten Stromtransport durch $\nabla_{\|} T$ zu gestatten. Das Potential fällt deshalb zur Kathode hin ab. Mit der steil ansteigenden Temperatur nimmt $\beta_{1}$ dann aber so stark zu, daß das Potential wieder ansteigen muß, um eine zu große Stromdichte zu verhindern. Im mittleren Bereich des Bogens verläuft das Tem- 


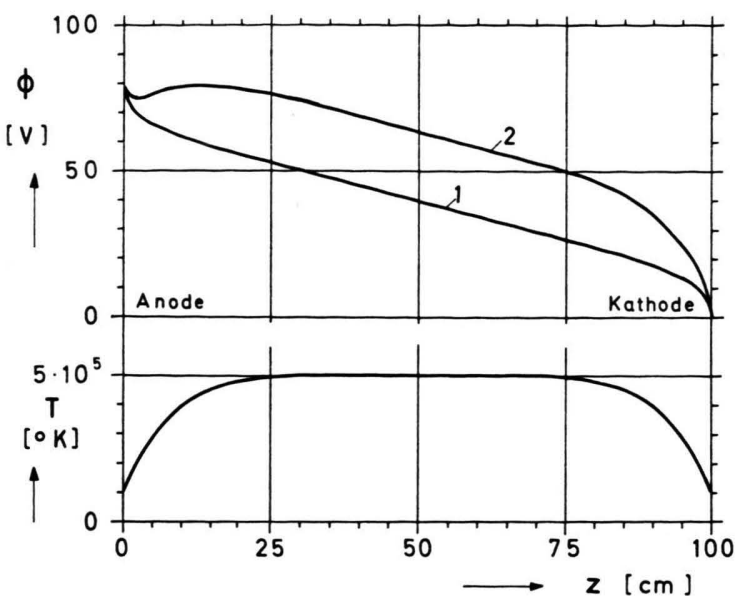

Abb. 11. Axiale Temperaturverteilung und axiale Potentialverteilungen bei $r=0$ ohne (1) und mit (2) Berücksichtigung von $\nabla_{\|} T$.

peraturprofil so flach, daß der Strom praktisch nur durch $E_{z}$ verursacht wird; die Potentialkurven 1 und 2 verlaufen deshalb parallel. Vor der Kathode muß $E_{z}$ den Strom gegen $\nabla_{\|} T$ treiben, die Kurve 2 fällt daher steiler ab als die Kurve 1. Diese Wechselwirkung von $\nabla \Phi$ und $\nabla T$, die - wie oben gezeigt - sogar zu einer Feldumkehr führen kann, wurde schon von verschiedenen Autoren an eindimensionalen Modellen für vollionisierte Plasmen unter Berücksichtigung der Energiebilanz untersucht ${ }^{13}, 14$.

\subsubsection{Der Einfluß des radialen Temperaturprofils auf $\Phi(r), E_{z}(r)$ und $j_{z}(r)$}

Das radiale Temperaturprofil beeinflußt den radialen Verlauf des Potentials $\Phi$ über den Hall-Parameter $\omega_{\mathrm{e}} \tau_{\mathrm{e}}$ und über die nichtelektrischen, stromtreibenden Kräfte $\left(\nabla_{\perp} T\right.$ und $\left.\nabla_{\perp} p_{\mathrm{e}}\right)$ im Ohmschen Gesetz. Zur Demonstration der verschiedenen Einflüsse wurde die Potentialverteilung für folgende Parameterwerte berechnet: $r_{\mathrm{K}}=0,75 \mathrm{~cm}, r_{\mathrm{A}}=2,25$ $\mathrm{cm}, r_{\mathrm{W}}=3 \mathrm{~cm}, L=50 \mathrm{~cm}, T_{\max }=1,5 \cdot 10^{5}{ }^{\circ} \mathrm{K}, T_{\mathrm{W}}$ $=10^{3}{ }^{\circ} \mathrm{K}, p_{\mathrm{A}}=10^{3} \mathrm{~N} / \mathrm{cm}^{2}, B_{0}=4$ Tesla, $I=2 \cdot 10^{3} \mathrm{~A}$. Die Temperaturverteilung soll nicht von $z$ abhängen, ihr radialer Verlauf mit $T_{\mathrm{e}}=T_{\max }$ nach Gl. (29) ist in Abb. 12 a dargestellt. Dieses Temperaturprofil und die angegebenen Daten entsprechen etwa dem Experiment von ZANKL ${ }^{2}$. Ebenfalls in Abb. $12 \mathrm{a}$ ist der Verlauf des Hall-Parameter $\omega_{\mathrm{e}} \tau_{\mathrm{e}}$ eingetragen.

13 L. S. Hall, Phys. Fluids 7, 425 [1964].

14 V. Boffi, V. Molinari u. D. E. PARKs, RT/FI (68) 7, Rom 1968.

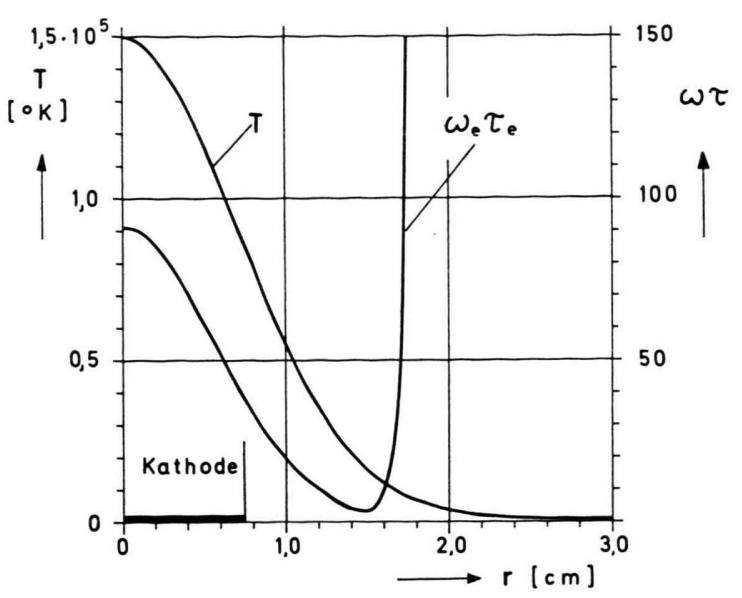

Abb. 12 a. Radiale Verteilungen von Temperatur $T$ und HallParameter $\omega_{\mathrm{e}} \tau_{\mathrm{e}}$.

Die Abb. 12 b zeigt radiale Potentialverteilungen bei $z=L / 2$, die unter verschiedenen Annahmen berechnet wurden. Allen Kurven gemeinsam ist der steile Anstieg von $\Phi$ auf das Potential der Anode bei $r=1,8-2 \mathrm{~cm}\left(T \leqq 7 \cdot 10^{3}{ }^{\circ} \mathrm{K}\right)$. Die Erklärung dieser Erscheinung, die schon von verschiedenen Autoren experimentell beobachtet wurde ${ }^{2,15,16}$, ist einfach zu geben. Für Temperaturen unterhalb von $7 \cdot 10^{3}{ }^{\circ} \mathrm{K}$ bei $p_{\mathrm{A}}=10^{3} \mathrm{~N} / \mathrm{cm}^{2}$ (스, 7,6 Torr) nimmt $\omega_{\mathrm{e}} \tau_{\mathrm{e}}$ so große Werte an $(>100)$, daß die elektrische Leitfähigkeit (in unserer Definition) senkrecht zum Magnetfeld viel kleiner ist als parallel $\left(\sigma_{2} / \sigma_{1}\right.$ $\left.<10^{-4}\right)$. Da die Anode wesentlich größer ist als die Kathode - wie bei allen zitierten Experimenten sind die Punkte außerhalb des Bogens durch eine Strecke parallel zu B mit der Anode verbunden, deren elektrischer Widerstand sehr viel kleiner ist als der des Weges zur Kathode, der immer Komponenten senkrecht zu B enthält. Diese extreme Spannungsteilung führt dazu, daß das kalte Gas praktisch das Potential der Anode annimmt.

Die Kurve 1 der Abb. 12 b zeigt den Potentialverlauf bei Berücksichtigung von $\nabla_{\perp} p_{\mathrm{e}}$ und $\nabla_{\perp} T$. Die bei $r=1,7 \mathrm{~cm}$ auftretende Spitze wird durch $\nabla \perp p_{\mathrm{e}}$ verursacht, wie die Rechnung unter Vernachlässigung dieses Effektes zeigt (Kurve 2). Es erscheint nicht sicher, daß die Spitze wirklich auftritt, da wir $\nabla_{\perp} p_{0}$ vernachlässigt haben, der eventuell zu einer Änderung von $\Phi(r)$ im schwach ionisierten Gas führen könnte. Wie der ähnliche Verlauf

15 I. Alexefr et al., High Pressure Arc in ORNL-4150.

16 R. SCHWENN, persönliche Mitteilung. 


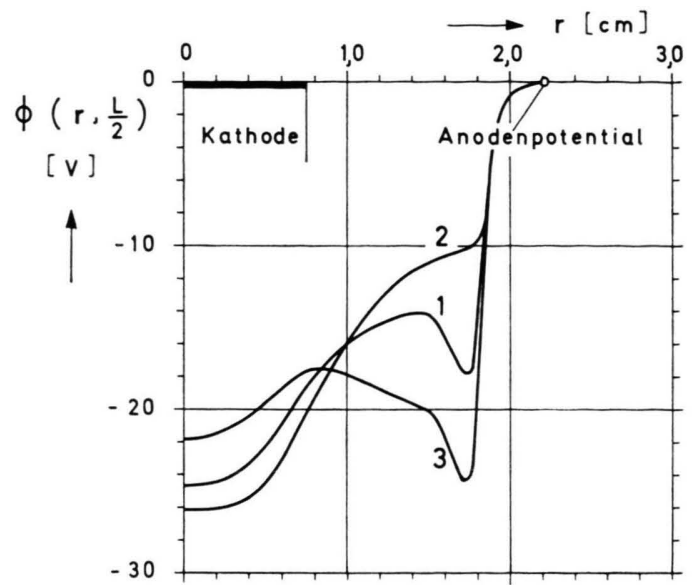

Abb. 12 b. Radiale Potentialverteilungen bei $z=L / 2$. Kurve 1: mit $\nabla_{\perp} p_{\mathrm{e}}$ und $\nabla_{\perp} T$; Kurve 2 : ohne $\nabla_{\perp} p_{\mathrm{e}}$; Kurve 3: ohne $\nabla_{\perp} T$.

der Kurven 1 und 2 bei kleinen Radien zeigt, beeinflußt $\nabla \perp p_{\mathrm{e}}$ den Potentialverlauf im vollionisierten Plasma nicht allzu stark. Sehr wesentlich ist dagegen der Einfluß von $\nabla_{\perp} T$, wie die Berechnung von $\Phi$ ohne Berücksichtigung von $\nabla_{\perp} T$ zeigt (Kurve 3). Zwischen $r=0,8 \mathrm{~cm} \quad\left(T=7,5 \cdot 10^{4}{ }^{\circ} \mathrm{K}\right)$ und $r=1,5 \mathrm{~cm}\left(T=1,5 \cdot 10^{4}{ }^{\circ} \mathrm{K}\right)$ ist die Steigung der Kurve 3 negativ, während die der Kurve 1 positiv ist. Dies zeigt, daß $\nabla \perp T$ einen nicht vernachlässigbaren elektrischen Strom radial nach außen treibt.

Der Einfluß von $\nabla \perp p_{\mathrm{e}}$ und $\nabla \perp T$ auf den Verlauf $E_{z}(r)$ ist relativ klein, wie der Vergleich der Kurven 1, 2 und 3 der Abb. 12 c zeigt. Der grundsätzliche Verlauf der drei Verteilungen ist gleich. Bei kleinen Radien ist $E_{z}$ praktisch konstant, dann folgt um $r=r_{\mathrm{K}}$ ein steiler Abfall auf etwa $60 \%$ des Achsenwertes. Die Ursache dafür ist die Größe des Hall-Parameters $\omega_{\mathrm{e}} \tau_{\mathrm{e}}$, der hier noch so groß ist, daß sich nach Gl. (23) eine Abklinglänge $r_{j}$ von etwa $1 \mathrm{~cm}$ ergibt. Der sich anschließenden Schulter im Verlauf von $E_{z}$ entspricht das ausgeprägte Minimum von $\omega_{\mathrm{e}} \tau_{\mathrm{e}}$ bei $T=1,6 \cdot 10^{4}{ }^{\circ} \mathrm{K} \quad\left(r_{j} \approx 15 \mathrm{~cm}\right)$. Schließlich fällt $E_{z}$ steil auf Null, da die Temperatur unter $10^{4}{ }^{\circ} \mathrm{K}$ sinkt, was $\omega_{\mathrm{e}} \tau_{\mathrm{e}}$ auf $10^{2}-10^{3}$ ansteigen läßt. Die Ursache liegt in der starken Abnahme der Stoßfrequenz der Elektronen. Dafür gibt es zwei Gründe:

a) Wegen der Abnahme des Ionisationsgrades stoßen die Elektronen nur noch selten mit Ionen, sondern fast ausschließlich mit neutralen Atomen zusammen, deren Stoßquerschnitt um mindestens zwei Größenordnungen kleiner ist als der CoulombQuerschnitt. b) Unterhalb von $10^{4}{ }^{\circ} \mathrm{K}$ fällt der Gesamtdruck steil auf den Kesseldruck $p_{\mathrm{A}}$ von einigen Torr $\mathrm{ab}$, da der Druckaufbau durch ambipolare Diffusion verschwindet ${ }^{6,7}$. Das führt zu einer weiteren $\mathrm{Zu}$ nahme von $\omega_{\mathrm{e}} \tau_{\mathrm{e}}$.

Diesen hohen Werten von $\omega_{\mathrm{e}} \tau_{\mathrm{e}}$ entspricht ein Verhältnis $\sigma_{2} / \sigma_{1}$ von $10^{-4}$ bis $10^{-6}$ und eine $\mathrm{Ab}$ klinglänge $r_{j}$ von wenigen Millimetern. Die Abb. $12 \mathrm{c}$ zeigt, daß $E_{z}$ tatsächlich über eine solche Strecke verschwindet und bestätigt damit das analytische

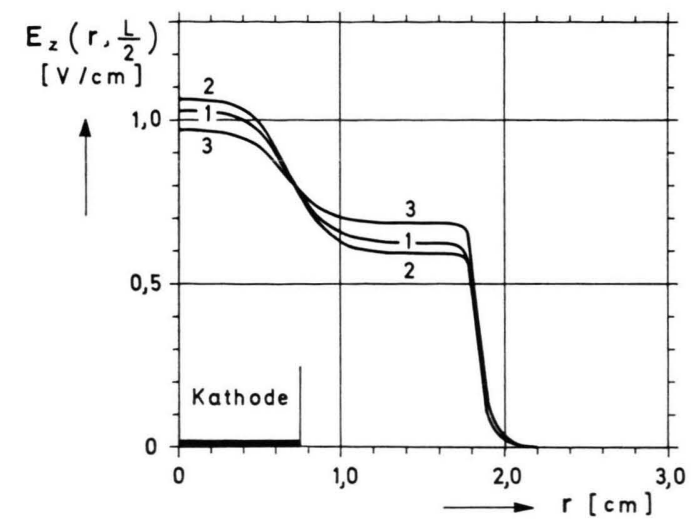

Abb. 12 c. Radiale Verteilungen von $E_{z}$ bei $z=L / 2$. Kurve 1: mit $\nabla_{\perp} p_{\mathrm{e}}$ und $\nabla_{\perp} T$; Kurve 2: ohne $\nabla_{\perp} p_{\mathrm{e}}$; Kurve 3: ohne $\nabla_{\perp} T$.

Resultat nach Abschn. 3.2, das eine markante Abnahme von $E_{z}$ bei $\sigma_{2} / \sigma_{1}=10^{-4}$ ergab (Abb. 5). Die Erscheinung, daß $E_{z}$ außerhalb des Bogens verschwindet, wurde auch schon experimentell beobachtet ${ }^{2,16}$. Die Abb. $12 \mathrm{~d}$ zeigt schließlich die Verteilungen der axialen Stromdichte $j_{z}$ mit und ohne Berücksichtigung von $\nabla \perp p_{\mathrm{e}}$ und $\nabla_{\perp} T$. Da wir das

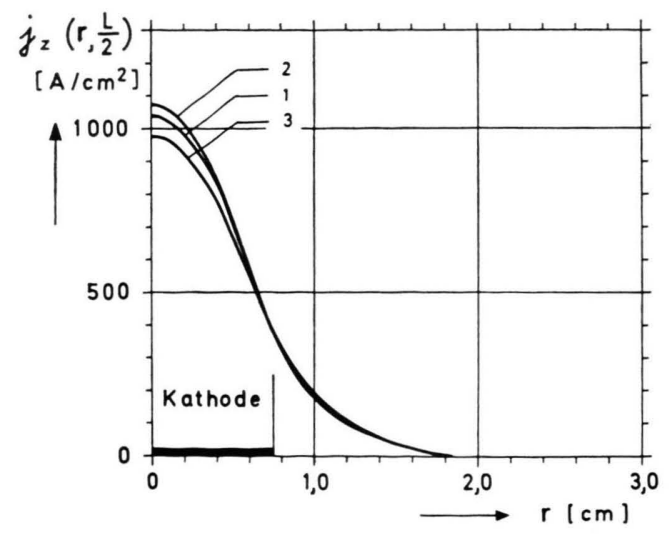

Abb. 12 d. Radiale Verteilungen von $j_{z}$ bei $z=L / 2$. Kurve 1: mit $\nabla_{\perp} p_{\mathrm{e}}$ und $\nabla_{\perp} T ;$ Kurve 2 : ohne $\nabla_{\perp} p_{\mathrm{e}}$; Kurve 3: ohne $\nabla \perp T$. 
Temperaturprofil festgehalten haben, sind die Unterschiede zwischen den verschiedenen Kurven durch die entsprechenden $E_{z}$-Verteilungen bestimmt und damit nicht groß.

\subsubsection{Der Einfluß des Kathodenradius auf $E_{z}(r)$}

In Abschn. 4.3.3 wurde gezeigt, daß $E_{z}$ um $r=r_{\mathrm{K}}$ steil abfällt, wenn $\omega_{\mathrm{e}} \tau_{\mathrm{e}}$ dort noch so groß ist, da $\beta$ die Abklinglänge $r_{j}$ nach Gl. (23) klein ist. Dieser Effekt wird einen wesentlichen Einfluß auf die Breite des Temperaturprofils und damit auf die Stabilisierung des Bogens haben, da die Ohmsche Heizung im wesentlichen proportional zu $E_{z}{ }^{2}$ ist. Aus der einfachen Vorstellung von Elektronen, die vom Magnetfeld auf dem Weg von der Kathode zur Anode geführt werden, kann man zwei wichtige Schlußfolgerungen ableiten:

a) Wenn die Radien von Anode und Kathode verschieden sind, wird sich bei ausreichend großem Hall-Parameter ein Stromdichteprofil einstellen, dessen Breite durch die kleinere Elektrode bestimmt wird. Bei den Experimenten ist das im allgemeinen die Kathode.

b) Entscheidend ist nicht der geometrische Radius der Kathode, sondern die Größe der emittierenden Fläche. Zieht sich der Stromansatz eines Bogens auf einen kleinen Brennfleck zusammen, so wird dieser die radiale Ausdehnung der Profile $\Phi(r), E_{z}(r)$ und $j_{z}(r)$ bestimmen.

Der Einfluß des Kathodenradius soll bei festgehaltenem Temperaturprofil nach Abb. 12 a demonstriert werden: $r_{\mathrm{K}}=0,75 ; 0,65 ; 0,55 \mathrm{~cm} ; r_{\mathrm{A}}=2,25$ $\mathrm{cm} ; r_{\mathrm{W}}=3 \mathrm{~cm} ; L=50 \mathrm{~cm} ; T_{\max }=T_{\mathrm{e}}=1,5 \cdot 10^{5}{ }^{\circ} \mathrm{K}$;

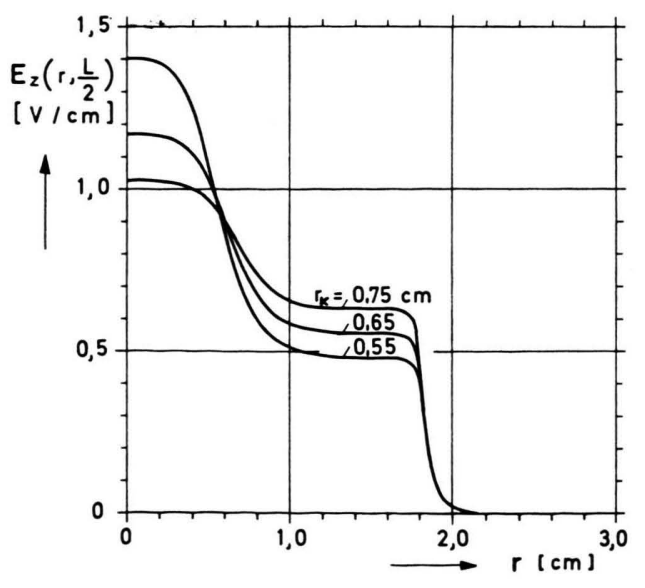

Abb. 13. Radiale Verteilungen von $E_{z}$ bei $z=L / 2$ für ein Temperaturprofil bei drei verschiedenen Kathodenradien.
$T_{\mathrm{W}}=10^{3}{ }^{\circ} \mathrm{K} ; p_{\mathrm{A}}=10^{3} \mathrm{~N} / \mathrm{cm}^{2} ; B=4$ Tesla $; I=$ $2 \cdot 10^{3}$ A. Die Abb. 13 zeigt $E_{z}(r)$ bei $z=L / 2$ für die drei verschiedenen Kathodenradien. Während $E_{z}$ bei $r_{\mathrm{K}}=0,75 \mathrm{~cm}$ auf $60 \%$ des Achsenwertes abfällt, sind es $35 \%$ bei $r_{\mathrm{K}}=0,55 \mathrm{~cm}$. Im ersten Fall wird die Ohmsche Heizung auf $36 \%$, im zweiten Fall auf $12 \%$ des Wertes bei $r=0$ reduziert. Tatsächlich ist der Effekt noch größer, da das Temperaturprofil bei $r_{\mathrm{K}}=0,55 \mathrm{~cm}$ schmaler sein wird als bei $r_{\mathrm{K}}=0,75 \mathrm{~cm}$.

Wegen des starken Einflusses des Kathodendurchmessers auf die radiale Breite der $E_{z}$-Verteilung kann man die Stromdichte in der Bogensäule bei einem vorgegebenen Gesamtstrom $I$ durch die Wahl des Kathodendurchmessers beeinflussen. Zur Erreichung möglichst großer Stromdichten und damit hoher Achsentemperaturen muß man den Durchmesser so klein wie nur irgend möglich wählen. Die Grenze wird dabei durch die Emissionsmechanismen und die thermische Belastbarkeit der Kathode gesetzt.

\subsubsection{Einfluß einer Rotation der Plasmasäule auf $E_{z}(r)$}

Experimente an Wasserstoffbögen mit hohlen, rohrförmigen Anoden haben gezeigt, daß eine starke Rotation der Plasmasäule mit Maximalgeschwindigkeiten von der Größenordnung $10^{6} \mathrm{~cm} / \mathrm{sec}$ auftreten kann, die durch $\boldsymbol{j} \times \boldsymbol{B}$-Kräfte angetrieben wird. Die Ursache sind Ströme, die innerhalb des Anodenrohres radial nach innen fließen ${ }^{17}, 18$. Um den Einfluß der Rotation auf $E_{z}(r)$ zu untersuchen, wurde die Potentialverteilung für folgende Annahmen und Parameterwerte berechnet:

a) Das von $z$ unabhängige radiale Profil der Rotationsgeschwindigkeit sei durch

$$
v_{\varphi}(r)=v_{\varphi \max } e\left(r / b_{v}\right)^{2} \exp \left(-r / b_{v}\right)^{2}
$$

gegeben.

b) Das Plasma sei homogen und habe die Temperatur $T=5 \cdot 10^{4}{ }^{\circ} \mathrm{K}$.

c) Die geometrischen Daten sind: $r_{\mathrm{K}}=1 \mathrm{~cm}$, $r_{\mathrm{A}}=3 \mathrm{~cm}, r_{\mathrm{W}}=4 \mathrm{~cm}, L=10 \mathrm{~cm}$.

d) $I=1,5 \cdot 10^{3} \mathrm{~A} ; B=1,4$ Tesla; $p_{\mathrm{A}}=10^{3} \mathrm{~N} / \mathrm{cm}^{2}$.

e) $\boldsymbol{v} \times \boldsymbol{B}$ weist radial nach außen.

17 C. MAhN, H. Ringler u. G. ZankL, Z. Naturforsch. 23 a, 874 [1968].

18 H. F. DöвELE, Z. Naturforsch. 24 a, 1249 [1969]. 
Die Abb. 14 zeigt das auf $v_{\varphi \max }$ normierte Profil der Rotationsgeschwindigkeit, die ihr Maximum bei $b_{v}=r_{\mathrm{K}}=1 \mathrm{~cm}$ annimmt. Der Ansatz $b_{v}=r_{\mathrm{K}}$ wird durch die Experimente gefordert. Ebenfalls dargestellt sind die berechneten Profile $E_{z}(r)$ etwa $1 \mathrm{~cm}$

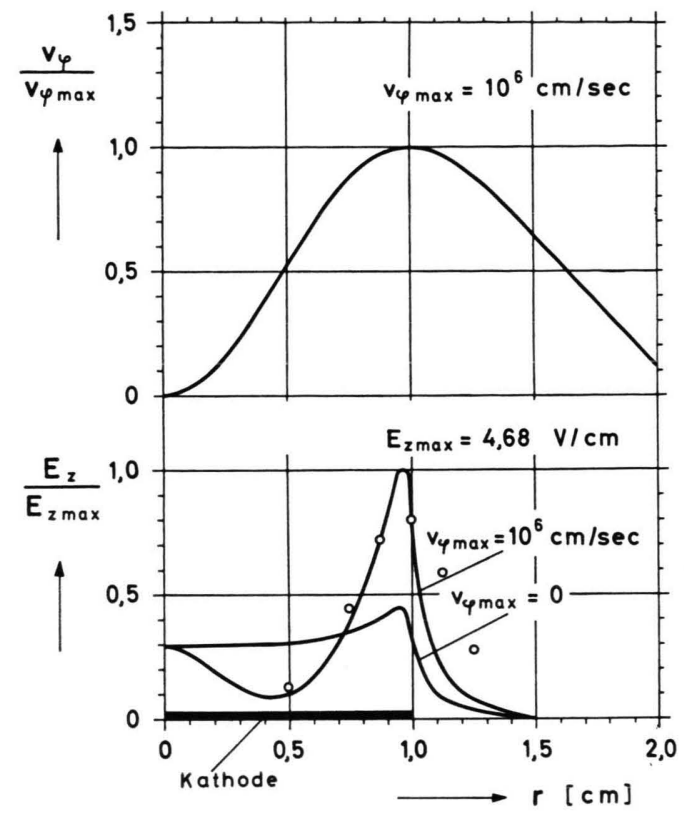

Abb. 14. Radiale Verteilung der Rotationsgeschwindigkeit mit entsprechender Verteilung von $E_{z}$. Kreise: experimentelle Werte nach ScHwenN ${ }^{16}$.

vor der Kathode für $v_{\varphi \max }=10^{6} \mathrm{~cm} / \mathrm{sec}$ und $v_{\varphi \max }$ $=0$. Im rotierenden Bogen tritt nach $\mathrm{Abb} .14$ ein steiles Maximum von $E_{z}$ bei $r=r_{\mathrm{K}}$ auf, das im wesentlichen nicht durch die kantige Kathodengeometrie verursacht wird, wie die Kontrollrechnung mit $v_{\varphi \max }=0$ zeigt. Bei Annäherung an die Anode verschwindet das Maximum von $E_{z}$ in zunehmendem Maße. Nur zum qualitativen Vergleich sind Meßergebnisse von SCHWENN ${ }^{16}$ eingetragen. Sein Experiment wird in etwa durch die Punkte a, c, d und e beschrieben. Einen Unterschied zwischen Theorie und Experiment bildet die Geometrie der Anode. Sie ist beim Experiment hohl und legt daher das Potential um die Achse der Anordnung nicht fest. Da $E_{z}$ nach Abb. 14 in Achsennähe klein ist, steigt das Potential bei $r=0$ nur wenig von der Kathode zur Anode hin an. Bei $r=r_{\mathrm{K}}$ nimmt das Potential viel stärker zu und endet beim Anodenpotential auf der Stirnfläche des Anodenrohres. Im Experiment kann sich deshalb eine rohrförmige Verteilung von $E_{z}$ längs der ganzen Säule ausbilden, während die
Theorie dies wegen der einfachen Randbedingungen nach Abb. 7 nicht zuläßt. Da das Plasma im wesentlichen um $r=r_{\mathrm{K}}$ geheizt wird, scheint es möglich, daß sich in einem kurzen, stark rotierenden Bogen eine Temperaturverteilung einstellen kann, die in der Bogenachse kleinere Werte annimmt als bei $r=r_{\mathrm{K}}$.

\section{Zusammenfassung}

Zur Erklärung der Stabilisierung eines Bogens im starken axialen Magnetfeld bietet sich die Vorstellung an, daß die von der Kathode emittierten Elektronen längs der magnetischen Feldlinien zur Anode wandern und dabei die Kraftlinien auf einer engen Bahn umkreisen. Nach diesem einfachen Bild ist der Durchmesser des stromführenden Kanals praktisch ebenso groß wie der Kathodendurchmesser oder im allgemeinen Fall gleich dem Durchmesser der kleineren Elektrode.

Wenn die Kathode nicht über ihre gesamte Oberfläche Elektronen emittiert, muß man offensichtlich den Kathodendurchmesser mit dem Durchmesser der emittierenden Fläche identifizieren. Dies scheint der einzige Punkt zu sein, an dem die Elektrodenmechanismen, die wir bei den Untersuchungen nicht berücksichtigt haben, einen Einfluß auf die Stromverteilung ausüben können. Eine starke Konzentration auf einen Brennfleck wird auch zu einer Konzentration der Stromverteilung in der Bogensäule führen.

Da die gyrierenden Elektronen gelegentlich mit anderen Teilchen zusammenstoßen und da das elektrische Feld der Bedingung $\nabla \times \boldsymbol{E}=0$ folgen muß, breitet sich der elektrische Strom auch in radialer Richtung aus. Die charakteristische Länge $r_{j}$ dieser Querdiffusion ist durch $r_{j}=L / \omega_{\mathrm{e}} \tau_{\mathrm{e}}$ gegeben (für $\left.\omega_{\mathrm{e}} \tau_{\mathrm{e}} \gg 1\right)$.

Da der Hall-Parameter $\omega_{\mathrm{e}} \tau_{\mathrm{e}}$ stark von der Temperatur abhängt, sind die Verteilungen von Temperatur und Potential eng miteinander gekoppelt. Werte von $\omega_{\mathrm{e}} \tau_{\mathrm{e}}$, die zu einer starken Reduzierung von $E_{z}$ führen, treten in zwei verschiedenen Temperaturbereichen auf. Bei hohen Temperaturen im vollionisierten Plasma kann der Hall-Parameter groß werden, da er sich wie $T^{5 / 2} / p_{\mathrm{e}}$ verhält. Bei Temperaturen zwischen $10^{4}$ und $2 \cdot 10^{4}{ }^{\circ} \mathrm{K}$ durchläuft $\omega_{\mathrm{e}} \tau_{\mathrm{e}}$ ein ausgeprägtes Minimum, um dann unterhalb von $10^{4}{ }^{\circ} \mathrm{K}$ wegen der Abnahme der Coulomb-Stöße wieder steil anzusteigen (Abb. 12 a). 
Eine Stabilisierung des Bogens durch das Magnetfeld liegt dann vor, wenn das radiale Temperaturprofil mit so großen Werten über den Radius der kleineren Elektrode hinausgreift, da $\omega_{\mathrm{e}} \tau_{\mathrm{e}}$ dort noch genügend hohe Werte annimmt. In dem speziellen Fall nach Abschn. 4.3.3 und Abb. $12 \mathrm{c}$ fällt $E_{z}$ zunächst auf etwa die Hälfte des Achsenwertes. Die Energiezufuhr wird dadurch schon stark reduziert, weil $E_{z}$ quadratische in die Ohmsche Heizung eingeht.

Bei den heute experimentell erreichten Temperaturen in Wasserstoffbögen haben die Terme proportional zu $\nabla p_{\mathrm{e}}$ und $\nabla T$ im Ohmschen Gesetz keinen großen Einfluß auf $E_{z}$ und damit auf die Stabilisierung des Bogens. Dagegen kann die Lorentz-Kraft $\boldsymbol{v} \times \boldsymbol{B}$ das radiale Profil stark ändern.

Vollständig kann die Frage nach der Art der Stabilisierung eines Wasserstoffbogens im axialen Magnetfeld nur beantwortet werden, wenn Potentialund Energiegleichung simultan gelöst werden. Dies soll in einer náchfolgenden Arbeit behandelt werden. Die beschriebenen Untersuchungen zeigen aber eindeutig, daß der Bogen im Magnetfeld stark durch die Elektroden stabilisiert wird. Die axiale elektrische Feldstärke im Bogen endlicher Länge ist keineswegs konstant über dem Radius, sondern ist im wesentlichen auf den durch die Elektrodenradien bestimmten Zylinder beschränkt, da das magnetisierte Plasma das elektrische Vakuumfeld stark modifiziert. Will man zur Erzeugung hoher Temperaturen eine große Stromdichte erzeugen, so muß man deshalb den Kathodenradius so klein wie möglich wählen.

Herrn Dr. KaRL Graf Finckenstern danken wir für seine Beratung bei der Aufstellung der Differenzengleichungen. Ganz besonders bedanken wir uns bei Fräulein H. MÜLlER für ihre vielfältige Unterstützung bei der Aufstellung von Rechenprogrammen. Diese Arbeit wurde im Rahmen des Vertrages zwischen dem Institut für Plasmaphysik und der Europäischen Atomgemeinschaft über die Zusammenarbeit auf dem Gebiet der Plasmaphysik durchgeführt.

\section{Anhang}

\section{Die wichtigsten Bezeichnungen}

$\begin{array}{llll}\Phi & \text { elektr. Potential } & T_{\max } & \begin{array}{c}\text { Maximaltempera- } \\ \text { tur im Bogen }\end{array} \\ \mathbf{E} & \text { elektr. Feldstärke } & & \text { Wandtemperatur } \\ \boldsymbol{j} & \text { elektr. Stromdichte } & T_{\mathrm{W}} & \text { Wam vor } \\ I & \text { Gesamtstrom } & T_{\mathrm{e}} & \text { Temperatur vor }\end{array}$

\begin{tabular}{|c|c|c|c|}
\hline B & magnet. Induktion & & den Elektroden \\
\hline $\boldsymbol{h}$ & $\begin{array}{l}\text { Einheitsvektor } \\
\text { zu B }\end{array}$ & $p_{\mathrm{A}}$ & $\begin{array}{l}\text { Gesamtdruck im } \\
\text { kalten Gas }\end{array}$ \\
\hline$B_{0}$ & $\begin{array}{l}\text { axiale Komponente } \\
\quad \text { von } B\end{array}$ & $p_{0}$ & $\begin{array}{l}\text { Druck der Neutral- } \\
\text { teilchen }\end{array}$ \\
\hline$\omega_{\mathrm{e}}$ & $\begin{array}{l}\text { Gyrofrequenz } \\
\text { der Elektronen }\end{array}$ & $\begin{array}{l}p_{\mathrm{e}} \\
r_{\mathrm{e}}\end{array}$ & $\begin{array}{l}\text { Elektronendruck } \\
\text { Elektrodenradius }\end{array}$ \\
\hline$\omega_{\mathrm{e}} \tau_{\mathrm{e}}$ & Hall-Parameter & & allgemein \\
\hline $\boldsymbol{v}$ & $\begin{array}{l}\text { Schwerpunkts- } \\
\text { geschwindigkeit }\end{array}$ & $\begin{array}{l}r_{\mathrm{K}} \\
r_{\mathrm{A}}\end{array}$ & $\begin{array}{l}\text { Kathodenradius } \\
\text { Anodenradius }\end{array}$ \\
\hline$T$ & Temperatur & $\begin{array}{l}r_{\mathrm{W}} \\
L\end{array}$ & $\begin{array}{l}\text { Gefäßradius } \\
\text { Elektrodenabstand }\end{array}$ \\
\hline
\end{tabular}

\section{Formelsammlung zum Ohmschen Gesetz}

(Alle Gleichungen gelten im MKSA-System)

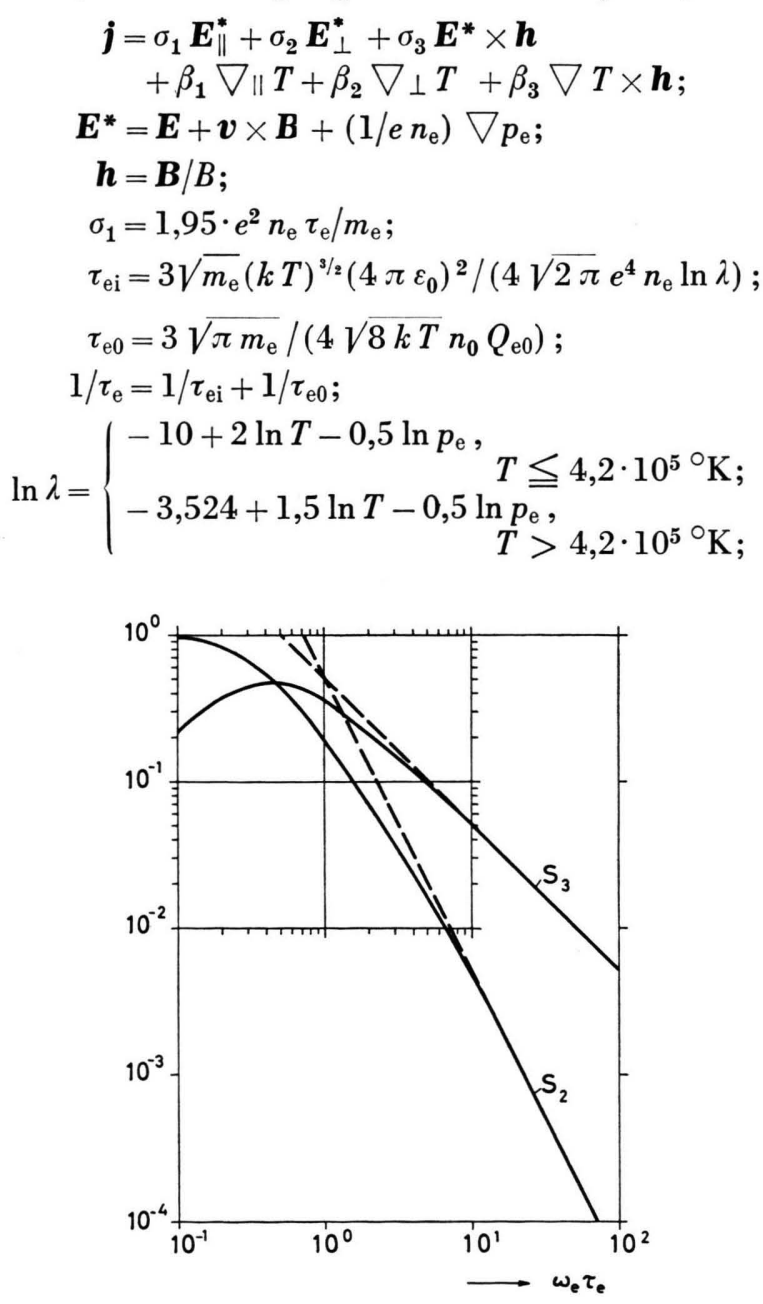

Abb. 15. Die Funktionen $S_{2}(x)$ und $S_{3}(x)$ zum Ohmschen Gesetz. Gestrichelte Gerade: asymptotische Verläufe für große Werte von $x$. 


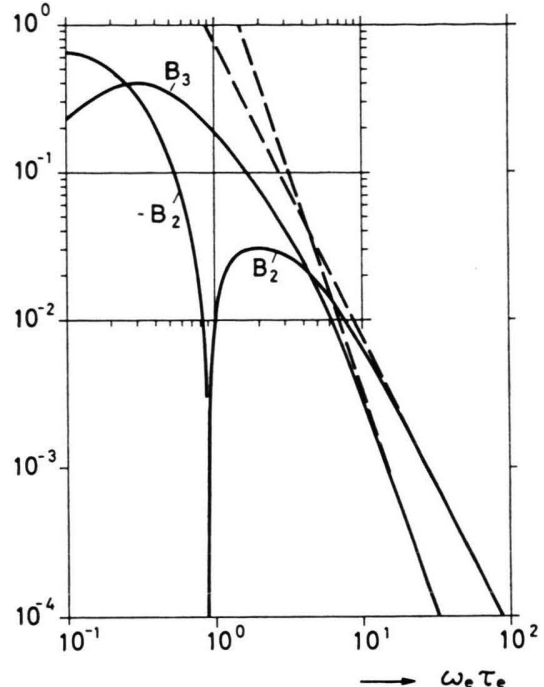

Abb. 16. Die Funktionen $B_{2}(x)$ und $B_{3}(x)$ zum Ohmschen Gesetz. Gestrichelte Gerade: asymptotische Verläufe für große Werte von $x$.

$$
\begin{gathered}
\sigma_{1}=1,512 \cdot 10^{-2} T^{3 / 2} / \ln \lambda[\mathrm{A} / \mathrm{Vm}], \quad T \text { in }{ }^{\circ} \mathrm{K} ; \\
x=\omega_{\mathrm{e}} \tau_{\mathrm{e}} ; \quad \sigma_{2}=\sigma_{1} S_{2}(x) ; \quad \sigma_{3}=-\sigma_{1} S_{3}(x) ; \\
\beta_{1}=0,711(k / e) \sigma_{1} ; \\
\beta_{2}=-(k / e) \sigma_{1} \mathrm{~B}_{2}(x) ; \\
\beta_{3}=-(k / e) \sigma_{1} B_{3}(x) ; \\
S_{3}(x)=(0,513 / N)\left(x^{8}+23,164 x^{6}+129,555 x^{4}\right. \\
\left.\quad+60,166 x^{2}+7,289\right) ; \\
S_{3}(x)=(0,513 / N)\left(x^{9}+31,284 x^{7}+252,266 x^{5}\right. \\
\left.\quad+129,480 x^{3}+17,154 x\right) ; \\
B_{2}(x)=(0,513 / N)\left(1,5 x^{8}+22,693 x^{6}+11,784 x^{4}\right. \\
\left.\quad-18,422 x^{2}-5,183\right) ; \\
B_{3}(x)=(0,513 / N)\left(6,601 x^{7}+102,431 x^{5}\right. \\
\left.\quad+95,895 x^{3}+18,101 x\right) ; \\
N= \\
\quad x^{10}+33,998 x^{8}+297,900 x^{6} \\
\quad+224,082 x^{4}+53,080 x^{2}+3,738 .
\end{gathered}
$$

Die Funktionen $S_{2}(x)$ und $S_{3}(x)$ sind in Abb. 15, $B_{2}(x)$ und $B_{3}(x)$ in Abb. 16 dargestellt. Ebenfalls eingetragen sind die asymptotischen Verläufe von $S_{2}(x), S_{3}(x), B_{2}(x)$ und $B_{3}(x)$ für $x \rightarrow \infty$ (gestrichelte Kurven). 